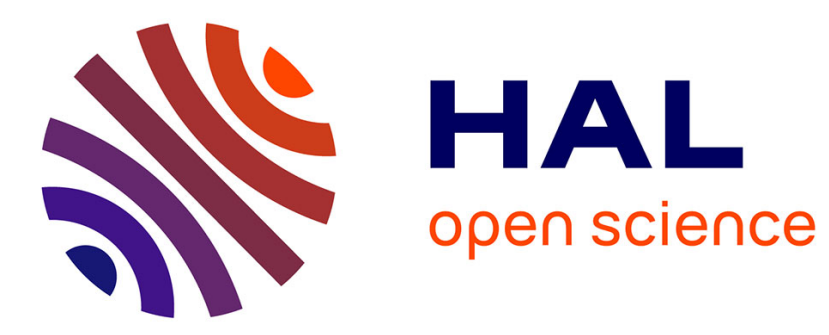

\title{
Silver-Gold Bimetallic Alloy versus Core-Shell Nanoparticles: Implications for Plasmonic Enhancement and Photothermal Applications
}

Rituraj Borah, Sammy W Verbruggen

\section{- To cite this version:}

Rituraj Borah, Sammy W Verbruggen. Silver-Gold Bimetallic Alloy versus Core-Shell Nanoparticles: Implications for Plasmonic Enhancement and Photothermal Applications. Journal of Physical Chemistry C, 2020, 124 (22), pp.12081-12094. 10.1021/acs.jpcc.0c02630 . hal-03348076

\section{HAL Id: hal-03348076 \\ https://hal.science/hal-03348076}

Submitted on 17 Sep 2021

HAL is a multi-disciplinary open access archive for the deposit and dissemination of scientific research documents, whether they are published or not. The documents may come from teaching and research institutions in France or abroad, or from public or private research centers.
L'archive ouverte pluridisciplinaire HAL, est destinée au dépôt et à la diffusion de documents scientifiques de niveau recherche, publiés ou non, émanant des établissements d'enseignement et de recherche français ou étrangers, des laboratoires publics ou privés. 


\title{
Silver-Gold Bimetallic Alloy versus Core-Shell Nanoparticles: Implications for Plasmonic Enhancement and Photothermal Applications
}

\author{
Rituraj Borah, ${ }^{\mathrm{a}, \mathrm{b}}$ Sammy W. Verbruggen ${ }^{\mathrm{a}, \mathrm{b} *}$ \\ ${ }^{a}$ Sustainable Energy, Air \& Water Technology (DuEL), Department of Bioscience Engineering, \\ University of Antwerp, Groenenborgerlaan 171, 2020 Antwerp, Belgium \\ ${ }^{\mathrm{b}}$ NANOlab Center of Excellence, University of Antwerp, Groenenborgerlaan 171, 2020 Antwerp, \\ Belgium \\ *Sammy.Verbruggen@uantwerpen.be
}

\begin{abstract}
Bimetallic plasmonic nanoparticles enable tuning of the optical response and chemical stability by variation of the composition. The present numerical simulation study compares Ag-Au alloy, Ag@Au core-shell, and Au@Ag core-shell bimetallic plasmonic nanoparticles of both spherical and anisotropic (nanotriangle and nanorods) shapes. By studying both spherical and anisotropic (with LSPR in the near-infrared region) shapes, cases with and without interband transitions of Au can be decoupled. Explicit comparisons are facilitated by numerical models supported by careful validation and examination of optical constants of Au$\mathrm{Ag}$ alloys reported in literature. Although both Au-Ag core-shell and alloy nanoparticles exhibit an intermediary optical response between that of pure $\mathrm{Ag}$ and $\mathrm{Au}$ nanoparticles, there are noticeable differences in the spectral characteristics. Also, the effect of the bimetallic constitution in anisotropic nanoparticles is starkly different from that in spherical nanoparticles due to the absence of Au interband transitions in the former case. In general, the improved chemical stability of $\mathrm{Ag}$ nanoparticles by incorporation of $\mathrm{Au}$ comes with a cost of reduction in plasmonic enhancement, also applicable to anisotropic nanoparticles with a weaker effect. A photothermal heat transfer study confirms that increased absorption by the incorporation of $\mathrm{Au}$ in spherical $\mathrm{Ag}$ nanoparticles also results in an increased steady state
\end{abstract}


temperature. On the other hand, anisotropic nanoparticles are inherently better absorbers, hence better photothermal sources and their photothermal properties are apparently not strongly affected by the incorporation of one metal in the other. This study of the optical/spectral and photothermal characteristics of bimetallic Au-Ag alloy versus core-shell nanoparticles provides a detailed physical insight for the development of new taylor-made plasmonic nanostructures.

\section{Introduction}

While the search for better plasmonic materials continues, most of plasmonic research so far has been based on Ag and Au nanostructures due to their excellent optical properties and ease of fabrication. ${ }^{1}$ Both elements have their own benefits and shortcomings. The low interband transition energy of $\mathrm{Au}(2.3 \mathrm{eV})$ leads to energy loss by such transitions, whereas the larger energy gap of $\mathrm{Ag}(3.7 \mathrm{eV})$ does not allow this interband transition, thus implying more efficient use of incident light energy for hot electron generation. ${ }^{2}$ Thus, while Ag suffers lower interband transition loss in the visible and near-infrared (NIR) range, Au is more resistant to oxidative degradation. For comparable sizes, Ag nanostructures have a Localized Surface Plasmon Resonance (LSPR) wavelength shorter than that of Au with stronger near field enhancement. ${ }^{3,4}$ Also, Ag nanoparticles have narrower and more symmetric plasmon absorption bands compared to $\mathrm{Au} .^{5}$ While starkly different in optical response as pure metal nanoparticles, bimetallic Ag-Au nanoparticles show the possibility to tune the optical features tailored to specific applications by regulating the composition and structure. ${ }^{6}$ Both core-shell and alloy nanoparticles of Ag-Au bimetallic systems have been shown to exhibit spectral characteristics that lay between those of pure Au and Ag nanoparticles. ${ }^{7}{ }^{10}$ Thus, bimetallic nanoparticles, constituted as both alloy and distinct core-shell phases, have been explored extensively, not just with $\mathrm{Ag}$ and $\mathrm{Au}$, but also using other metals such as $\mathrm{Cu}$, Pt and $\mathrm{Pd}$, amongst others. ${ }^{11}$ Considerable efforts have been devoted towards controlled synthesis of both core-shell and alloy nanoparticles with desired compositions. Generally, wet chemical synthesis of Au@Ag core-shell nanoparticles is straightforward and proceeds by the controlled reduction of an $\mathrm{Ag}$ precursor in the presence of $\mathrm{Au}$ nanoparticles, or seeds, that facilitate crystallization of $\mathrm{Ag}$ as a shell around the Au cores. ${ }^{12}$ Conversely, 
$\mathrm{Ag} @ \mathrm{Au}$ core-shell nanoparticles are difficult to synthesize in a similar way. In the presence of $\mathrm{Ag}$ nanoparticles, $\mathrm{Au}^{3+}$ ions trigger a galvanic replacement reaction that results in hollow structures. ${ }^{13}$ It has been shown that by controlling the relative kinetics of the galvanic replacement and the reduction by a reducing agent, growth of Au shells on silver nanoparticles can be achieved with smooth morphology. ${ }^{14-16}$ While a number of studies has reported direct synthesis methods for Ag-Au alloy nanoparticles by coreduction of $\mathrm{Ag}$ and Au precursors, ${ }^{8,}{ }^{17}$ recent work shows that traditional co-reduction methods do not necessarily lead to fully homogeneous alloys. ${ }^{18}$ Conversion of a hybrid Ag-Au nanostructure such as coreshell nanoparticles to alloys simply by heat treatment at temperatures as low as $250^{\circ} \mathrm{C}$ has also been shown to be a promising and facile synthesis route. ${ }^{19-21}$ The intrinsic aspect that sets alloy nanoparticles apart from bimetallic core-shell nanoparticles is the overlapping of energy bands of the constituting elements in the alloy. Thus, the distinct plasmonic behavior of alloy nanostructures results from the altered dielectric properties of the mixed material. ${ }^{22}$ In contrast, core-shell nanoparticles exhibit plasmonic behavior that is a hybrid of the individual plasmon modes of the core and the shell. ${ }^{23}$

This intrinsic difference and the ease of switching from core-shell to alloy naturally raises a question: Which is the better plasmonic material, alloy or core-shell? The answer clearly is subject to the intended application. In general, gold nanoparticles are preferred over silver nanoparticles for photothermal applications due to higher stability and absorption intensity of gold. ${ }^{24,}{ }^{25}$ Espinosa et al. recently showed that silver nanoparticles lose their photothermal properties with time due to chemical degradation inside human mesenchymal stem cells; but with a thin Au shell surrounding the Ag core, the nanoparticles can be stabilized completely. ${ }^{24}$ On the other hand, spectral tunability of Ag-Au alloy nanoparticles enables wider coverage of the visible light spectrum for plasmon-assisted photocatalysis. ${ }^{4}$ Similarly, the bimetallic constitution facilitates spectral tunability for SERS-based sensing. ${ }^{26,}{ }^{27}$ While spectral tunability is an advantage, plasmonic hot carrier generation is strongly dependent on the nanoparticle composition. ${ }^{2}$

In the present work, a comparative simulation study is performed on the plasmonic characteristics of $\mathrm{Au}-$ Ag bimetallic alloy and core-shell nanoparticles, and includes both spherical and anisotropic (nanorods and 
nanotriangles) shapes. The resulting photothermal effect of these nanoparticles is also evaluated and compared. In particular, absolute absorption/scattering spectra, near-fields and local temperature fields are studied to create a deeper physical insight into how the nanoparticle constitution can be perfected and optimally tuned to a given plasmonic application.

\section{Problem Specification and Numerical Methodology}

The present study involves electromagnetic simulations of isolated bimetallic spherical and anisotropic nanoparticles with a core-shell configuration as well as an alloy configuration of $\mathrm{Ag}$ and Au. Figure 1 shows schematics of spherical nanoparticles with corresponding Trasmission Electron Microscopy (TEM) images and Energy Dispersive X-Ray (EDX) analysis maps reproduced from literature. Since scattering is low for small nanoparticles $(<50 \mathrm{~nm})$, the size of the nanoparticles in this work was fixed at $60 \mathrm{~nm}$, so that both absorption and scattering are significant over the entire range of studied compositions in order to draw meaningful comparisons. Similarly, the studied nanotriangles in this work are of $60 \mathrm{~nm}$ each side and $7 \mathrm{~nm}$ thickness, and the nanorods are $20 \mathrm{~nm}$ in diameter and $80 \mathrm{~nm}$ in length. The implications for smaller nanoparticles are discussed briefly in the later sections for the completeness of the results. 
(a)

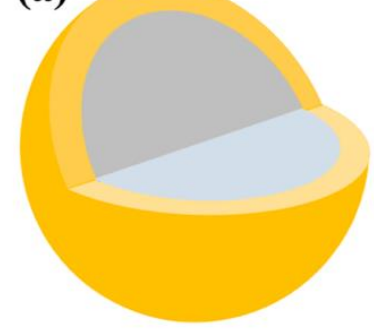

Ag@Au core-shell

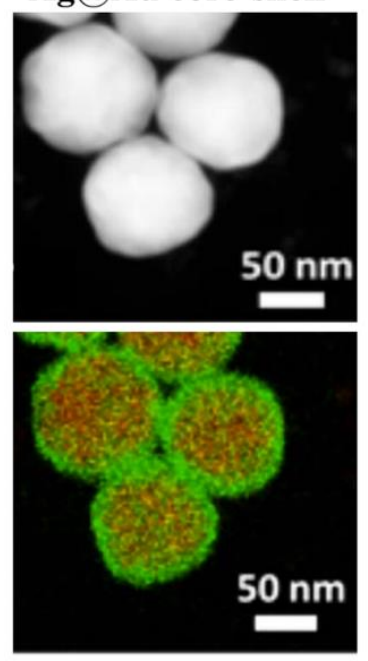

(b)

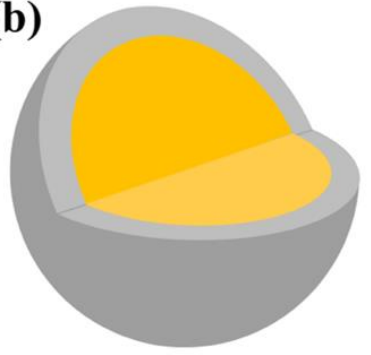

Au@Ag core-shell
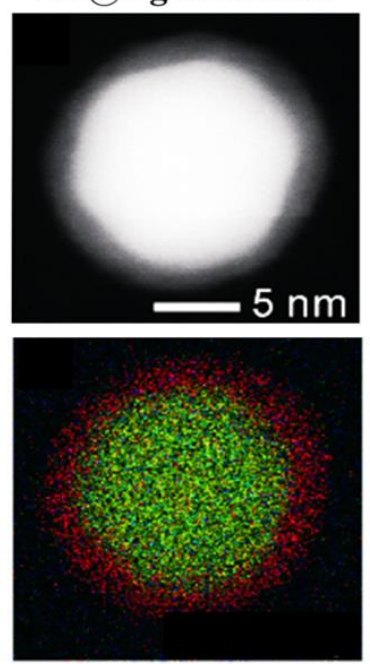

(c)

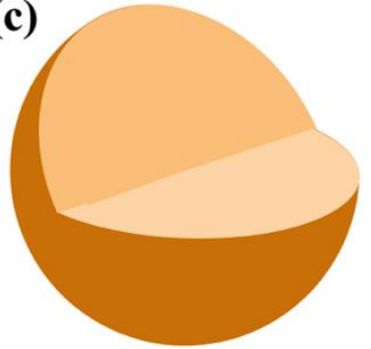

Au-Ag Alloy
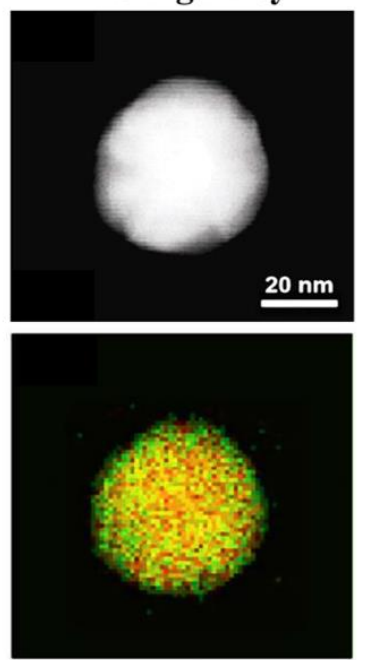

Figure 1. Schematic of spherical bi-metallic Au-Ag nanoparticles as (a) Ag@Au core-shell, (b) Au@ Ag core-shell and (c) alloy with their TEM images and EDX mappings taken from references ${ }^{15} 1217$. The images were adapted and reproduced with permission from American Chemical Society (C) 2017), IOP Publishing (C 2012), Wiley-VCH (C) 2019).

An FEM-based numerical framework COMSOL Multiphysics was implemented to solve the frequency domain form of Maxwell's equations:

$\nabla \times\left(\mu_{r}^{-1} \nabla \times E_{s c}\right)-k_{o}^{2}\left(\varepsilon_{r}-j \frac{\sigma}{\omega \varepsilon_{o}}\right) E_{s c}=0$

In equation (1), $\mu_{r}, \varepsilon_{r}$, and $\sigma$ denote material properties namely relative permeability, relative permittivity and electrical conductivity respectively. The scattered field solution of equation (1) is obtained in terms of the local scattered electric field, $E_{s c}$. The total field $E$ is the superposition of the scattered and incident electric fields, $E_{s c}$ and $E_{i n c}$ respectively. A spherical computational domain was implemented with the 
nanoparticle at the center and a perfectly matched layer surrounding the domain. The perfectly matched layer was discretized by prismatic elements, while the computational domain including the nanoparticle was discretized by tetrahedral elements. While for plain spherical nanoparticles this discretization scheme has been proven to work well, ${ }^{28}$ to ensure that it is also valid for the core-shell nanoparticles with a shell thickness as thin as $2.14 \mathrm{~nm}$, a grid independence test was carried out. Comparing a 199043 element grid (minimum element size: $1.08 \mathrm{~nm}$, maximum element size: $25 \mathrm{~nm}$ ) with a 594120 element grid (minimum element size: $0.144 \mathrm{~nm}$, maximum element size: $14.4 \mathrm{~nm}$ ) in Figure $\mathrm{S} 4$ makes it clear that even distinctly different grid geometries provide excellent agreement in the spectra. Similar grid independence tests are also shown for a nanotriangle and a Ag@Au nanocube with a $0.5 \mathrm{~nm}$ thin shell, Figure S4. This grid independence tests validate the accuracy of the present numerical results.

The optical cross-sections were obtained from the scattered field solution. Absorption and scattering crosssections for a nanoparticle can be defined as:

$$
\begin{gathered}
\sigma_{a b s}=\frac{W_{a b s}}{I} \\
\sigma_{s c}=\frac{W_{s c}}{I}
\end{gathered}
$$

Where $W_{a b s}$ and $W_{s c}$ are energy absorbed and scattered per unit time by the nanoparticle respectively, and $I$ is the intensity of incident light.

$I=\frac{1}{2} c \varepsilon\left|E_{\text {inc }}\right|^{2}$

Now, the power absorbed and scattered in eq. (2) is obtained from the numerical solution as follows:

$$
W_{a b s}=\frac{1}{2} \iiint_{V} \operatorname{Re}\left[(\sigma E+j \omega D) \cdot E^{*}+j \omega B \cdot H^{*}\right] d V
$$


$W_{s c}=\frac{1}{2} \oiint_{s} \operatorname{Re}\left[E_{s c} \times H_{s c}^{*}\right] \cdot n d S$

Where, the superscript $*$ and $D$ stand for complex conjugate and displacement currents respectively. The integration in eq. (5) is throughout the nanoparticle volume and the surface integral in eq. (6) is over the nanoparticle surface. The built-in functions in COMSOL Multiphysics were used for these computations.

The heat transfer analysis for the evaluation of the photothermal effect of nanoparticles in a water medium requires solving the steady state form of the heat equation: ${ }^{29,30}$

$\rho C_{P} U . \nabla T=k \nabla^{2} T+q$

Here, $U$ and $T$ are the velocity vector and temperature, respectively. While, $k, \rho$, and $C_{p}$ denote material properties namely, thermal conductivity, density and specific heat capacity. In eq. (7), $q$ is the rate of heat generation per unit volume due to absorption obtained as in eq. (5). For a given set values of these parameters, the problem is essentially simplified to the numerical solution of the conduction equation, as the velocity vector $U$ in eq. (7) for the convective velocity of water can be neglected since convection will not be significant for an object of extremely small dimensions, also indicated by a very small Rayleigh number (in the order of $10^{-14}$ ). ${ }^{31}$ While the convection does not influence the development of the temperature field, the investigation of the convective velocities, however small they are, is also important for applications in micro-environments. This aspect is not included in this work. A suitably large domain is chosen that imitates an infinite medium. In practice, the boundaries have to be extremely far as the temperature spatial decay scales with $1 / r$, where $r$ is the distance from the nanoparticle. ${ }^{32}$ A spherical domain of $2000 \mathrm{~nm}$ radius was found to be suitable as an infinite medium. The thermophysical properties were taken from relevant literature sources. ${ }^{33}, 34$ Since the thermal conductivity of water is significantly low as compared to $\mathrm{Au}$ and $\mathrm{Ag}$, the variability of the thermal conductivity of $\mathrm{Au}$ and $\mathrm{Ag}$ with temperature does not impact the conductive heat transfer characteristics. This is also implicit from the low value of the Biot number meaning the lump capacitance approximation is valid for both of the cases. 


\section{Results and Discussion}

\subsection{Model Validation}

First, the present numerical model was validated using past experimental results. For alloy nanoparticles, the literature on reliable optical constant data is scent. Also, not all of the handful of such studies cover a wide spectral range or, more importantly, can reproduce experimental results accurately. ${ }^{35,}{ }^{36}$ Reliance on weighted average values as shown in some past literature is a too simplistic approach as the energy of the transitions changes with the composition. ${ }^{8,9,37}$ In view of the literature available on this topic, Rioux et al. provides a rigorous model based on critical point analysis of the band structure of Au and Ag to show close agreement between theoretical and experimental values. ${ }^{22}$ Their model overcomes the unphysicality of the simple Drude-CP (critical point) model reported by Etchegoin et al. ${ }^{38,39}$ inspite of the good quality of fit obtained. In this model the summation of Lorentzian oscillators representing individual (interband) transitions in the Drude-Lorentz model has been replaced by an integration over of the joint density of states (jDOS), so that the interband transition part of the dielectric function is a convolution of the jDOS with a simple Lorentzian oscillator whose strength is energy-dependent. Thus, the shape of the dielectric function is determined by the shape of the jDOS which is depedent on the critical points i.e., Van Hove singularities in the jDOS. The two functions CP1 and CP2 defined by Rioux et al. for the interband transitions were obtained by the approximation of the joint density of states (jDOS). Upon defining this 10 parameter model of $\mathrm{Ag}$ and $\mathrm{Au}$, the composition dependence of each parameter in the alloy has been modeled as a secondorder polynomial giving rise to 3 sub-parameters. Thus, a 30 parameter model combined with the Drude free electron term was obtained for the composition depedent dielectric function of an Ag-Au alloy that overcomes the shortcomings of the previous literature. For the fitting of this model to experimental data, they implemented an algoritm suited for multi-parameter optimization based on least square error.

Nonetheless, it is still useful to compare the values reported in different studies, as presented in Figures S1, S2 and S3.22, $40-{ }^{42}$ For Ag-Au alloys, the agreement of Nishijima et al. and Peña-Rodríguez et al. with Rioux et al. is considerably better than Gong et al. over the visible light range, Figure S3. Further 
assessment of these data is done by comparing computed normalized scattering cross section spectra with experimental results reported by Patskovsky et al. in Figure 2(a).$^{43}$ Clearly, optical constants predicted by the theoretical model of Rioux et al. work well in estimating the extinction spectra of both pure as well as alloy nanoparticles. Au generally shows a better agreement than Ag using optical constants from different literature sources, as illustrated in Figures S5 and S6. When compared for $60 \mathrm{~nm}$ pure Ag nanoparticles, optical constants from Palik ${ }^{44}$ appear to give large deviations from the rest, Figure S5. Also, the sharp quadrupolar peak in the absorption cross section is absent when optical constants from Palik were used. Apart from the experimental errors in the optical constants, the deviation of the computed spectral peak position from the experimental one, may also arise from the sensitivity to the surrounding environment, morphological irregularity of nanoparticles, instrumental errors and so on. ${ }^{45}$ In Figure 2(b), computed extinction spectra using the dielectric constants of Rioux et al. are compared with experimental UV-Vis spectra of Ag nanocubes with a thin shell of Au reported by Yang et al. ${ }^{46}$ The simulated results match the experimental data both quantitatively and qualitatively. With this overview of the available literature on the optical constants of $\mathrm{Au}$ and $\mathrm{Ag}$ alloy, it is reasonable to proceed with the model of Rioux et al. as it estimates the constants for any arbitrary composition and the agreement with experiments is shown to be the most satisfactory. 

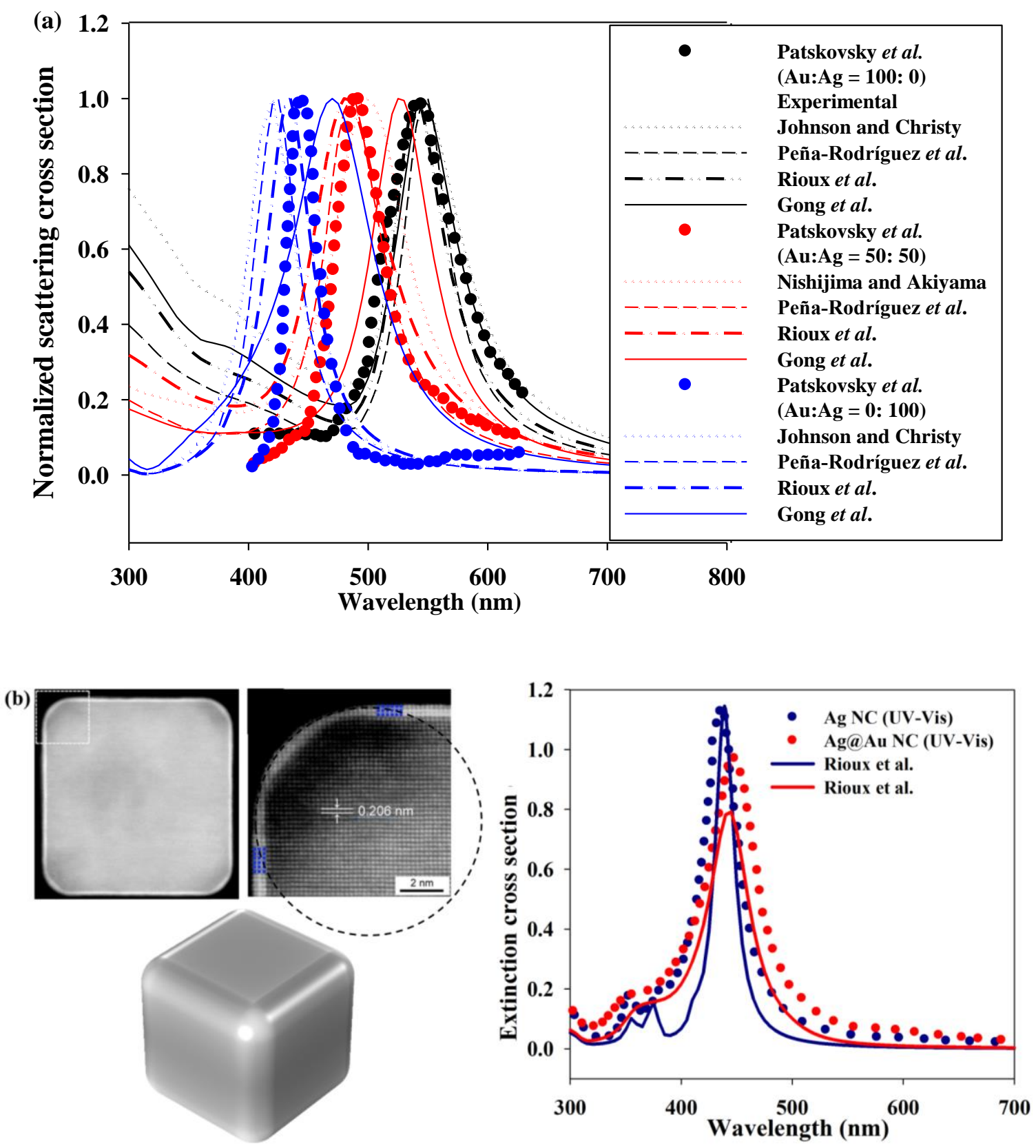

Figure 2. (a) Comparison of experimental scattering spectra of Patskovsky et al. ${ }^{43}$ with computed spectra for optical constants from different literature sources: Johnson and Christy ${ }^{47}$, Peña-Rodríguez et al. ${ }^{41}$, Rioux et al. ${ }^{22}$ and Gong et $a l .{ }^{42}$ for pure $\mathrm{Au}$, pure Ag and 50:50 alloy Au:Ag (Note: Optical constants from Peña-Rodríguez et al. ae for 48:52 molar ratio of $\mathrm{Au}$ to $\mathrm{Ag}$ ). Color codes: black for $\mathrm{Au}: \mathrm{Ag}=100: 0$, red for $\mathrm{Au}: \mathrm{Ag}=50: 50$, blue for $\mathrm{Au}: \mathrm{Ag}=0: 100$ (b) TEM images of $\mathrm{Ag}$ nanocubes $(\mathrm{AgNC})$ with thin $\mathrm{Au}$ protecting layer ${ }^{46}$, visualization of 3D nanocube model in the present 
study, comparison of experimental extinction spectra with calculated spectra with constants from and Rioux et al. The TEM images are adapted and reproduced from American Chemical Society (@ 2014).

\subsection{Comparison of Plasmonic Properties: spherical nanoparticles}

\subsubsection{Absorption and scattering behavior}

Before delving into bi-metallic nanoparticles, it is useful to analyze the role of the interband transitions and free electrons that eventually result in plasmonic excitation. As mentioned earlier, Au introduces such interband transitions, while Ag can be approximated as an entirely free electron contributor in the visible range. While the free elctrons result in plasmonic excitation, with consequent radiative and non-radiative damping, the interband transition contributes to "non-plasmonic" light capture and consequent thermalization. Discrimintating between both effects is possible by using a mathematical model for the wavelength dependent dielectric constants, where the parameters concerning individual effects can be selectively dropped. For this, the Drude-CP model that exploits critical point (CP) analysis ${ }^{38,39}$ has been shown to fit well with the dielectric constants of Au reported in Johnson and Christy ${ }^{47}$, Figure 3(a) and (b). Figures 3(c) and (d) compare optical spectra obtained by separately considering the interband transitions (CP terms) and the free electrons (Drude term) in the dielectric constants with the spectrum that is obtained by considering the complete dielectric function. The position and the intensity of the LSPR is an outcome of these two effects coupled together. For a $60 \mathrm{~nm}$ nanosphere, the Drude free electron part in the absence of interband transitions yields plasmonic excitation that results in large radiative damping (scattering) and small non-radiative damping (absorption) at the LSPR. It is important to note that this purely plasmonic excitation is strongly dependent on nanoparticle's shape and size. In presence of the interband transition, however, the LSPR is red-shifted significantly, and the scattering component is drastically reduced with a proportionate enhancement in the absorption. Thus for $60 \mathrm{~nm}$ Au nanoparticles, the absorption cross section has a significant contribution from the interband transitions. However, whether it is the dominant contributor cannot be concluded directly. In contrast, the small size of a $20 \mathrm{~nm}$ nanoparticle facilitates strong non-radiative damping of the plasmons contributing to the absorption cross section far more 
significantly than interband transitions, Figure 3(e) and (f). Thus, while in the absence of interband transitions, the plasmonic excitation is remarkably strong as evidenced by the sharp absorption and scattering peaks; taking interband transition into account results in a drastic drop of the plasmonic excitation and the consequent optical cross sections. These competing effects of free electrons and interband transitions combined with the size and shape factors will also determine the optical response of Ag-Au bimetallic nanoparticles, as will be shown further. 

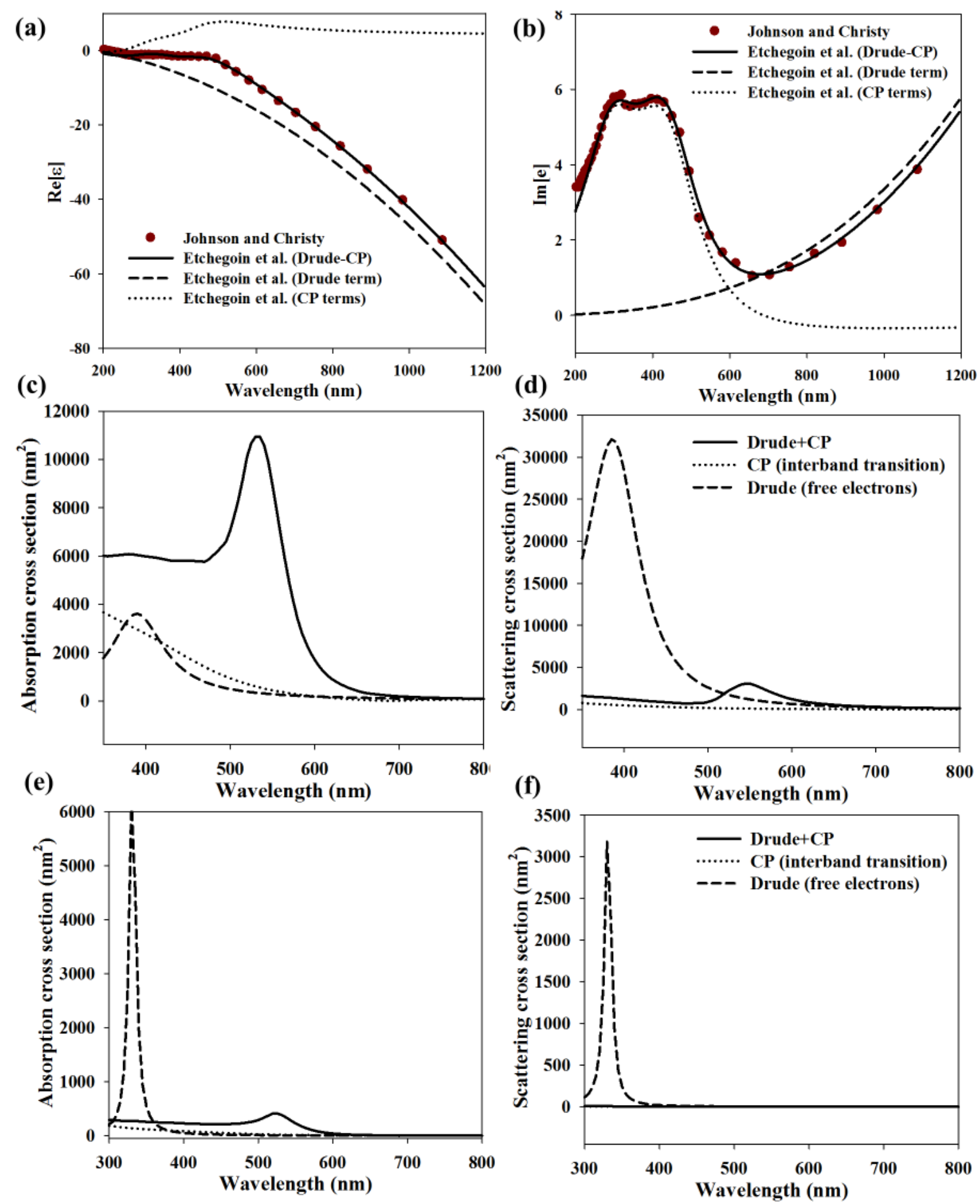

Figure 3. (a) Comparison of Johnsona and Christy ${ }^{47}$ dielectric constants for Au with Drude-CP model as reported by Etchegoin et al ${ }^{38,39}$. The dielectric functions only considering the free electron Drude term and the interband transition $\mathrm{CP}$ term are also shown. (c, d) Comparison of absorption and scattering cross sections of a $60 \mathrm{~nm}$ Au nanoparticle 
with those calculated considering the Drude term and the CP terms alone. (e, f) Comparison of absorption and scattering cross sections of a $20 \mathrm{~nm}$ Au nanoparticle with those calculated considering the Drude term and the CP terms alone.

Figures 4 to 6 compare spherical Au-Ag alloy nanoparticles, Ag@ Au core-shell nanoparticles, and Au@ Ag core-shell nanoparticles, respectively, all of $60 \mathrm{~nm}$ in diameter and for different compositions. The general trends of the bimetallic constitution are discussed first before delving into the specific cases. In general, incorporation of $\mathrm{Au}$ in $\mathrm{Ag}$ nanoparticles induces a red shift of the plasmon band accompanied by a reduction in scattering, and increase in absorption as seen for both alloy and core-shell structures. From the discussion related to Figure 3, it can be attributed to the interband transitions in Au. Incorporation of even $20 \% \mathrm{Au}$ significantly increases the absorption cross section for both alloy and core-shell nanoparticles accompanied by a corresponding drastic reduction in the scattering component. It is useful to note that the absorption component of the total energy extinction dissipates thermally while the scattered part is re-radiated to the surroundings. The absorption in Au is contributed by interband transitions from the $d$-band and plasmon decay, both resulting in hot carrier generation that eventually thermalize if not utilized in some other way. ${ }^{48,}$ ${ }^{49}$ Zheng et al. showed that hot carriers from plasmon decay are more energetic than those from interband transitions and are able to cross a $\sim 1 \mathrm{eV}$ Shottky potential barrier more efficiently. While in silver, interband transitions are absent. ${ }^{50}$ The resulting absorption and scattering components are a cummulative effect of these mechanisms and radiative damping of the plasmons. A $60 \mathrm{~nm} \mathrm{Ag}$ nanoparticle is characterized by a strong radiative decay of plasmons (large scattering component) and weak thermalization. Incorporation of $\mathrm{Au}$ in $\mathrm{Ag}$ consequently introduces new interband transitions and reduction of the plasmonic excitation of the pure $\mathrm{Ag}$ state. The net result is an increase of the absorption component and a decrease in scattering. The resulting decrease in the overall extinction intensity upon introduction of $\mathrm{Au}$ in $\mathrm{Ag}$ is also clear from Figure S7. Importantly, this effect of interband transition is not seen in the case of anisotropic nanoparticles as will be discussed later. The change of optical intensities with the amount of Au incorporated does not seem to follow a continuously increasing trend. While for alloy nanoparticles, it can be attributed to the 
changes of the optical properties with different alloy compositions; for core-shell nanoparticles, it is rather attributed to the coupling of different sizes of the core and the shell. ${ }^{23}$
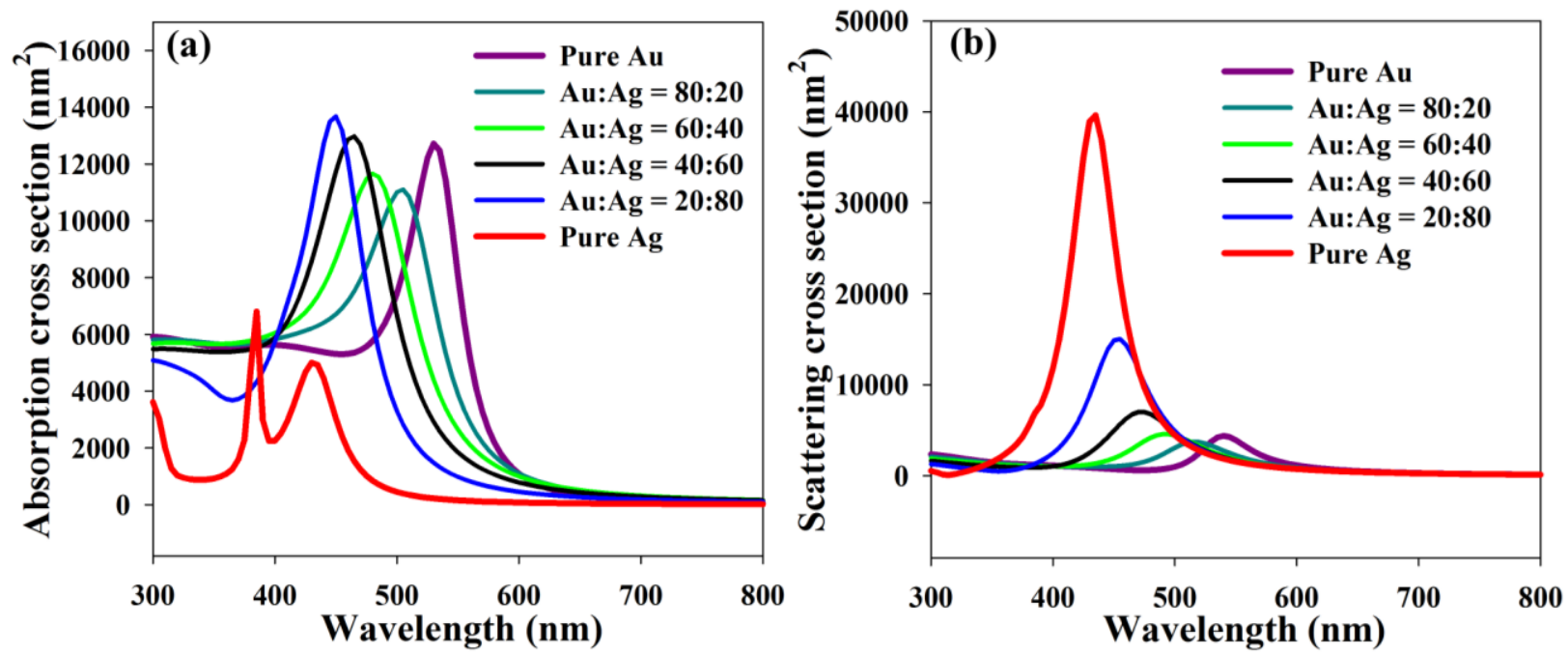

Figure 4. Absorption and scattering cross section of Ag-Au alloy nanoparticles with varying composition and fixed size of $60 \mathrm{~nm}$.
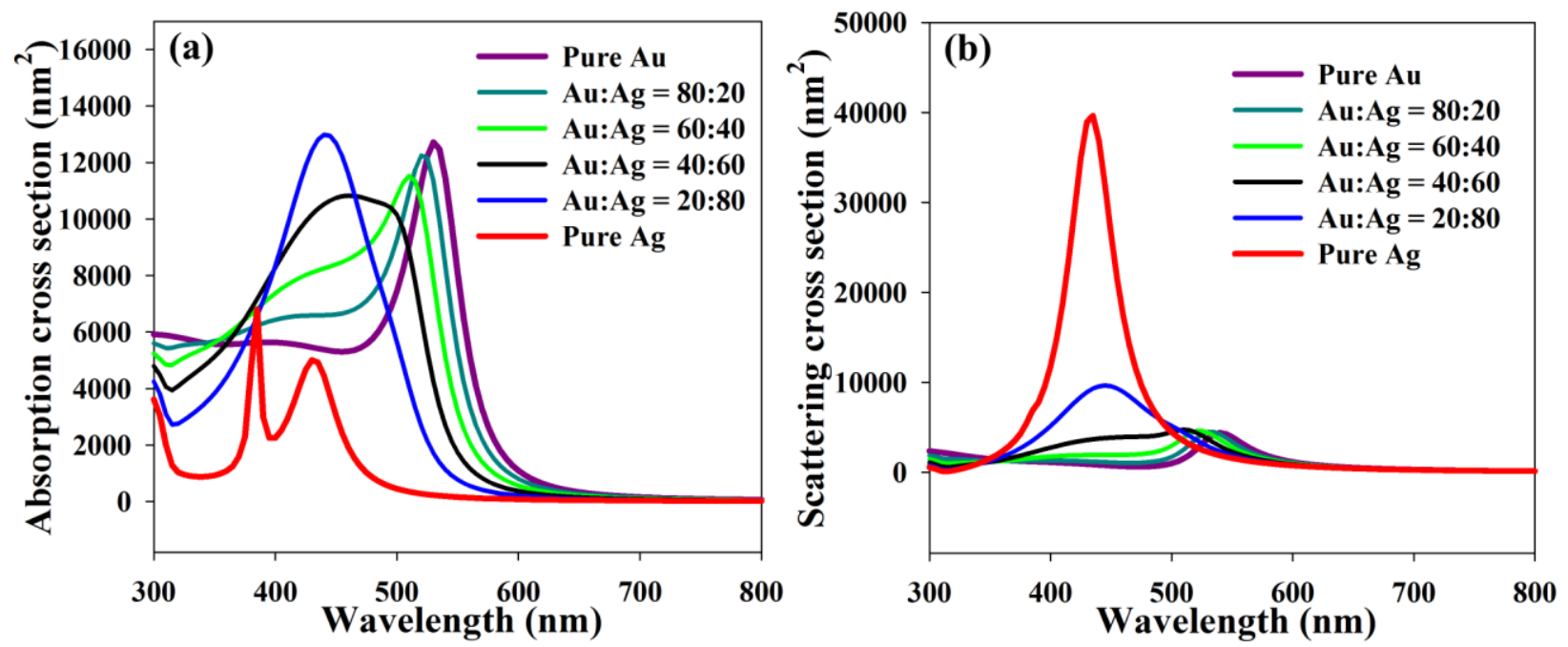

Figure 5. Absorption and scattering cross section of $\mathrm{Ag} @ \mathrm{Au}$ core-shell nanoparticles with varying composition and fixed size of $60 \mathrm{~nm}$. 

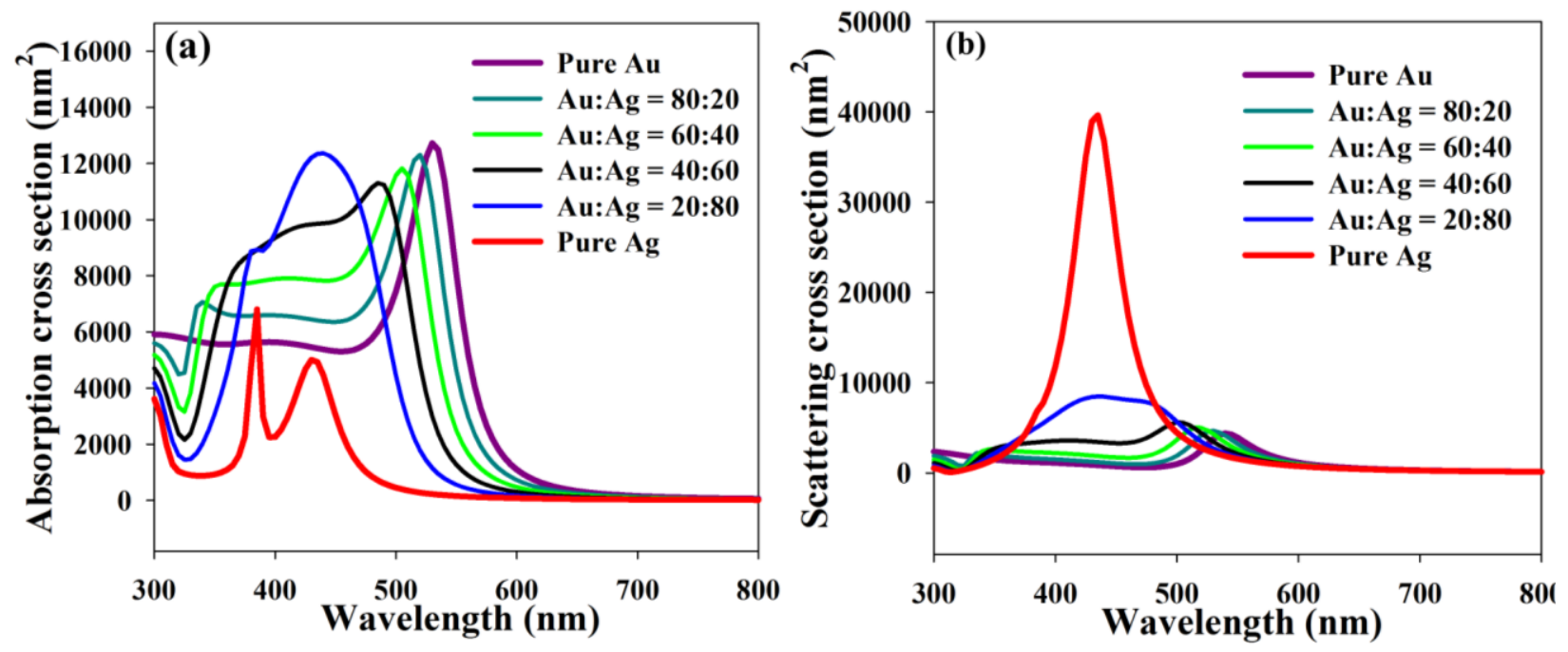

Figure 6. Absorption and scattering cross section of Au@ Ag core-shell nanoparticles with varying composition and fixed size of $60 \mathrm{~nm}$.

Further comparison of alloy with core-shell constitutions shows that the optical spectra of the alloy nanoparticles, Figure 4, are generally sharper (with one characteristic LSPR peak) than the core-shell ones, Figures 5 and 6. Experimental evidence of these trends has been reported in literature by means of UV-Vis data of bimetallic nanoparticles. ${ }^{51,52}$ The asymmetric broadening of the LSPR peaks of core-shell nanoparticles with different intermediate $\mathrm{Ag:Au}$ compositions, shown in Figure 5 and 6 , is also in agreement with experimental observations for Au@Ag core-shell nanoparticles. ${ }^{53}$ - 55 According to plasmon hybridization theory, the hybrid plasmon modes constituted by individual plasmon modes of core and shell are strongly dependent on the core and shell sizes. Thus, the optical spectra result from these hybrid modes. In contrast, an alloy nanoparticle has a homogeneous composition with its optical properties determined by the composition. Alloys exhibit optical properties that are intermediary between pure $\mathrm{Ag}$ and $\mathrm{Au} .{ }^{56,57,9,10}$ It is noteworthy that recent research on $\mathrm{Au}-\mathrm{Ag}$ alloy nanoparticles has shown that similar single dipolar LSPR peaks are also obtained for inhomogeneous bimetallic nanoparticles that attain a core-shell-like architecture. ${ }^{17}$ These differences in the spectral characteristics between core-shell and alloy are well supported by experimental results. ${ }^{53}$ 
Considering the use of bimetallic nanoparticles for the tuning of the LSPR wavelength by variation of the composition, it is useful to understand the dependence of the spectral shift on the composition for both alloy and core-shell configurations. As apparent from Figure 7, the spectral shift upon introduction of $\mathrm{Au}$ in $\mathrm{Ag}$ is considerably different for all three configurations, $\mathrm{Ag} @ \mathrm{Au}, \mathrm{Au} @ \mathrm{Ag}$ and alloy, taking into consideration the dipolar resonance peak. For alloy nanoparticles, the red-shift is proportionate to the amount of Au as alloy when moving from pure $\mathrm{Ag}$ to $\mathrm{Au}$, also supported experimentally. ${ }^{10,8}$ For core-shell nanoparticles, the red-shift is not gradual. For instance, in Figure 7(a), going from 40\% to 60\% Au in the Ag@ Au coreshell nanoparticle, the red shift of the absorption spectra is significantly larger than that while going from $60 \%$ to $80 \%$. Also core-shell nanoparticles have irregular and wider spectra. For both absorption and scattering components in Figure 7(a) and (b), the LSPR peaks for the Au @ Ag core-shell nanoparticle are located at larger wavelengths than for the alloy nanoparticles of the same overall composition.
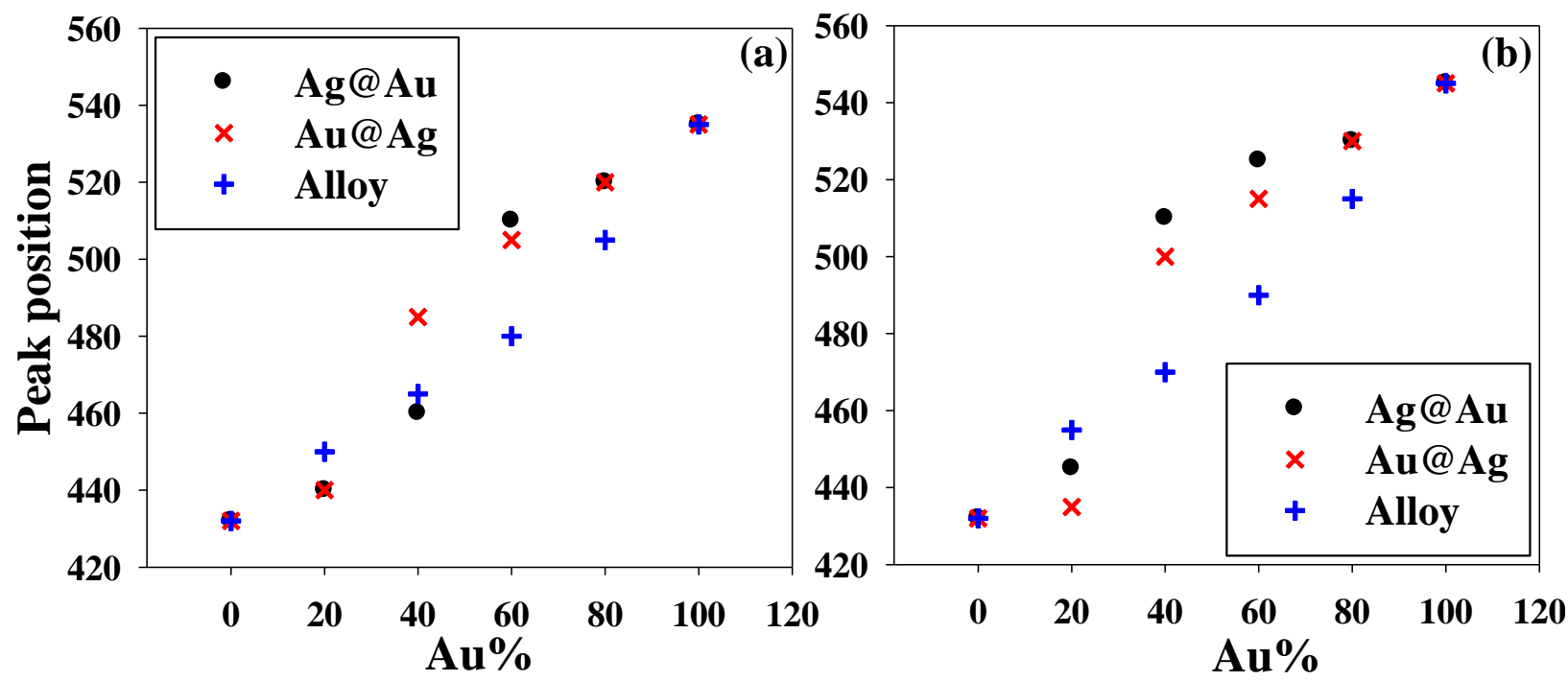

Figure 7. Spectral shift of absorption (a) and scattering (b) cross section with incorporation of Au for Ag@Au, $\mathrm{Au} @ \mathrm{Ag}$ and alloy nanoparticles of $60 \mathrm{~nm}$ in diameter.

\subsubsection{Near-field enhancement}

Despite the reduction of plasmon induced hot carriers upon introduction of $\mathrm{Au}$ in $\mathrm{Ag}$, the chemical stabilization of $\mathrm{Ag}$ by $\mathrm{Au}$ is often quite useful. The result of energy consumption by interband transition is 
reduced plasmonic excitation. Zheng et al. quantified the hot carrier generation by plasmon decay separated from that by interband transitions and showed good correspondence with experiments. ${ }^{48}$ Generally for spherical nanoparticles, the near-field enhancement is always weaker in gold nanospheres than in silver due to the contribution by interband transitions, Figure S8. Figure 8 shows that addition of $20 \% \mathrm{Au}$ in $\mathrm{Ag}$ also significantly weakens the near-field enhancement around the thus-obtained alloy or core-shell nanoparticles when compared to the near-field enhancement of pure $\mathrm{Ag}$ nanoparticles. The alloy nanoparticle does not seem to be significantly different from the core-shell particle in terms of the attenuation of the near-field enhancement. Thus, stabilization of Ag nanoparticles in atmospheric environment by adding a thin Au shell has strong consequences with respect to the plasmonic properties.

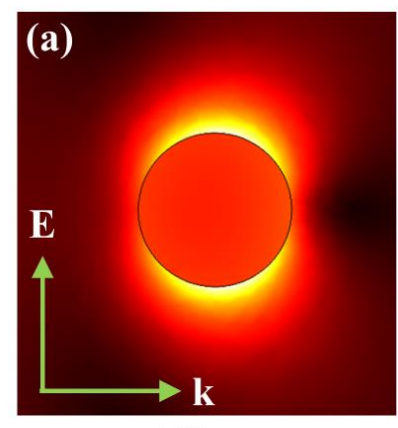

$435 \mathrm{~nm}$

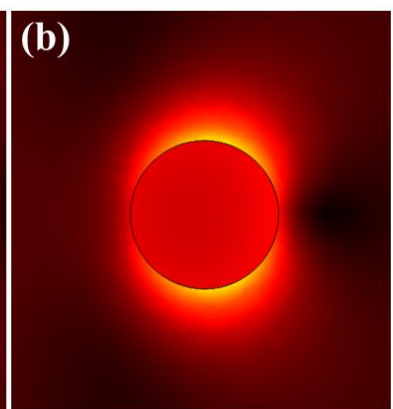

$450 \mathrm{~nm}$

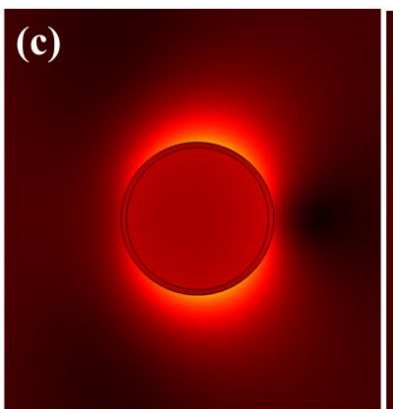

$440 \mathrm{~nm}$

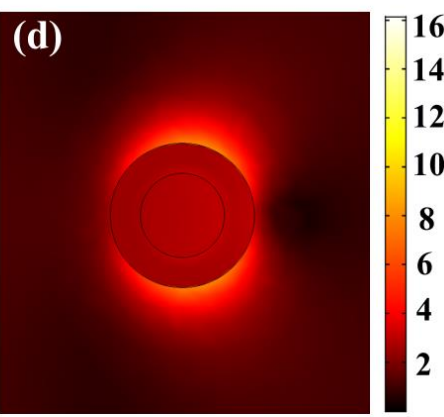

$440 \mathrm{~nm}$ Incident Wavelength

Figure 8. Near field enhancement with respect to the incident radiation around (a) pure Ag nanoparticle and (b) alloy (c) $\mathrm{Ag} @ \mathrm{Au}$ (d) $\mathrm{Au} @ \mathrm{Ag}$ nanoparticles of molar composition Au:Ag = 20:80 at their respective dipolar plasmon resonance modes.

Importantly, while $20 \%$ Au addition significantly alters both the spectral and near-field characteristics of a pure Ag nanoparticle, the thin shell of $\mathrm{Au}$ around $\mathrm{Ag}$ is only $2.14 \mathrm{~nm}$ in that case. Since the preceding comparisons were for a constant $60 \mathrm{~nm}$ nanoparticle size, incorporation of $\mathrm{Au}$ as the shell material entails a corresponding reduction of the Ag core size. To show that the drastic reduction in scattering and increase in absorption is not due to the shrinking of the core, optical spectra for a $60 \mathrm{~nm} \mathrm{Ag}$ nanoparticle with a 
$2.14 \mathrm{~nm} \mathrm{Au}$ shell around it (total diameter: $64.28 \mathrm{~nm}$ ) were evaluated, Figure 9. Clearly, the effect of a slightly shrunk core is insignificant compared to that of the addition of the thin shell, resulting in strong changes in the optical spectra for a $2.14 \mathrm{~nm}$ thick shell surrounding a $60 \mathrm{~nm}$ particle core. The resulting optical response of the $64.28 \mathrm{~nm}$ nanoparticle differs from that of the $60 \mathrm{~nm}$ nanoparticle primarily due to the overall enlargement of the particle size.
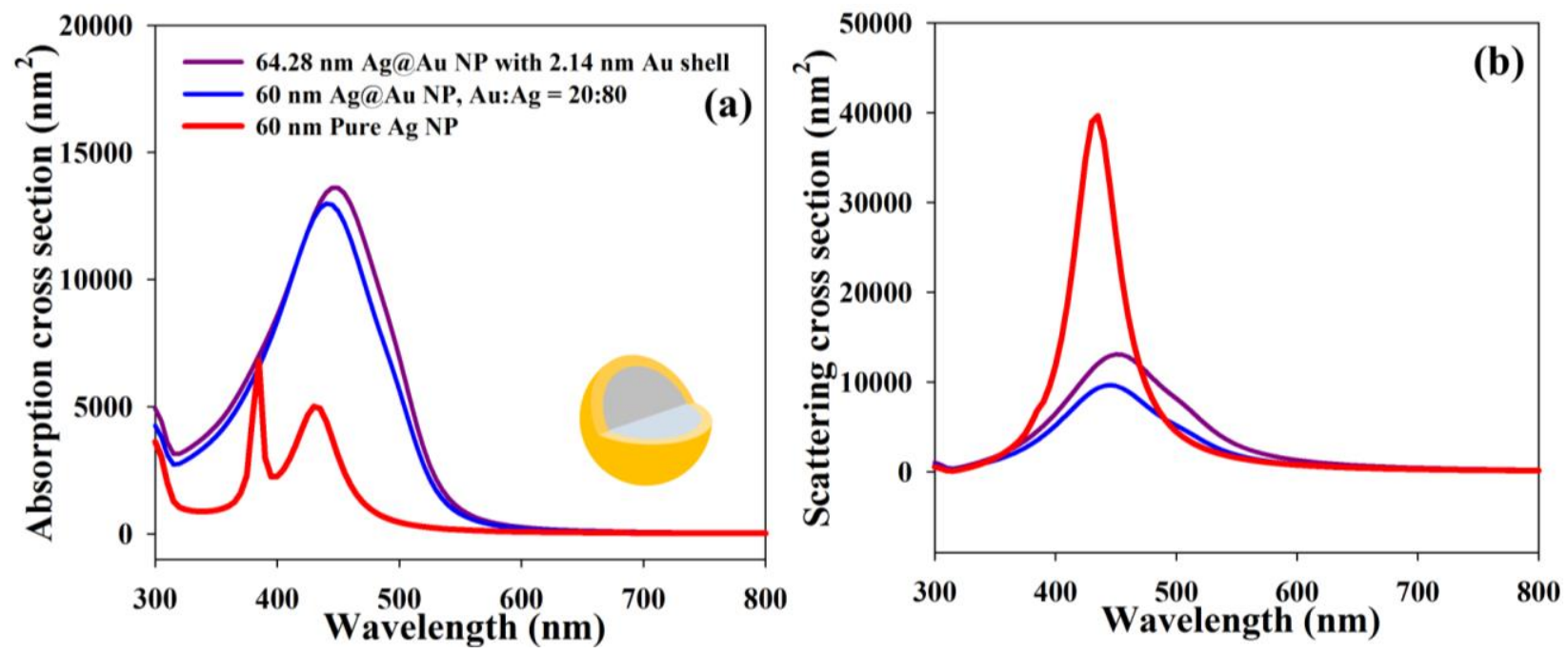

Figure 9. Effect of extra $2.14 \mathrm{~nm}$ thick Au shell on a $60 \mathrm{~nm}$ Ag nanoparticle compared to Au incorporation as shell (2.14) in a $60 \mathrm{~nm}$ nanoparticle for 20:80 molar composition of Au:Ag. (a) Absorption cross section (b) scattering cross section.

\subsection{Comparison of Optical Properties: anisotropic nanoparticles}

\subsubsection{Absorption and scattering behavior}

The discussion so far concerned nanospheres for which the LSPR wavelength falls in the wavelength range in which Au exhibits interband transitions. Thus, it is interesting to investigate the effect of bimetallic constitutions when the LSPR is in the near-infrared or infrared region where Au does not have interband transitions. In that regard, synthesis of anisotropic nanoparticles such as nanorods and nanotriangles, which exhibit LSPR in the near-infrared and infrared region, has seen considerable progress in the recent years. ${ }^{58}$ 
In continuation of the preceding discussion, the effect of incorporating $20 \% \mathrm{Au}$ in an anisotropic $\mathrm{Ag}$ nanoparticle as a shell or alloy on the optical response is shown in Figure 10 and Figure 11. In this case the LSPR is located in the near-infrared and infrared region. The effect of addition of $20 \% \mathrm{Au}$ in $\mathrm{Ag}$ in this case is interestingly different from the behavior that was observed in the case of nanospheres. In the absence of interband transitions, the absorption with the consequent thermalization is only caused by plasmon decay. Thus, apart from the obvious shift in plasmon band position, both the LSPR absorption and scattering intensities of an Ag nanorod, Figure 10, and a nanotriangle, Figure 11, are not significantly altered after adding an Au shell. Interestingly, when Au is added as alloy, the absorption is reduced a bit more, but the scattering is reduced dramatically. This rather strong change in the plasmonic response can be attributed to the altered band structure of the alloy. Experiments comparing the plasmonic hot carrier generation in $\mathrm{Ag} @ \mathrm{Au}$ core-shell and alloy nanorods or nanotriangles would thus provide useful insight into these observations. Regarding the spectral shift, while the Ag@Au core-shell nanorods and nanotriangles both exhibit a blue shift, in the case of alloying only the nanotriangles shows a blue shift but not the alloy nanorods. At this point it is also important to inspect the effect of corner sharpness changes even by addition of a thin layer, which for nanotriangles is obviously significant. Figure 12(a) and (b) show that layer by layer uniform enlargement of nanotriangles smoothens the corners leading to a blue shift of the spectra accompanied by an increase in both absorption and scattering. While for nanorods, adding uniform layers results in a usual red shift due to elongation and further remains unaffected by any edge effect, Figure 12(c, d). Thus, it is clear that the edge smoothening also has a significant role in the LSPR blue shift of nanotriangles when $20 \% \mathrm{Au}$ is added as shell or alloy. 

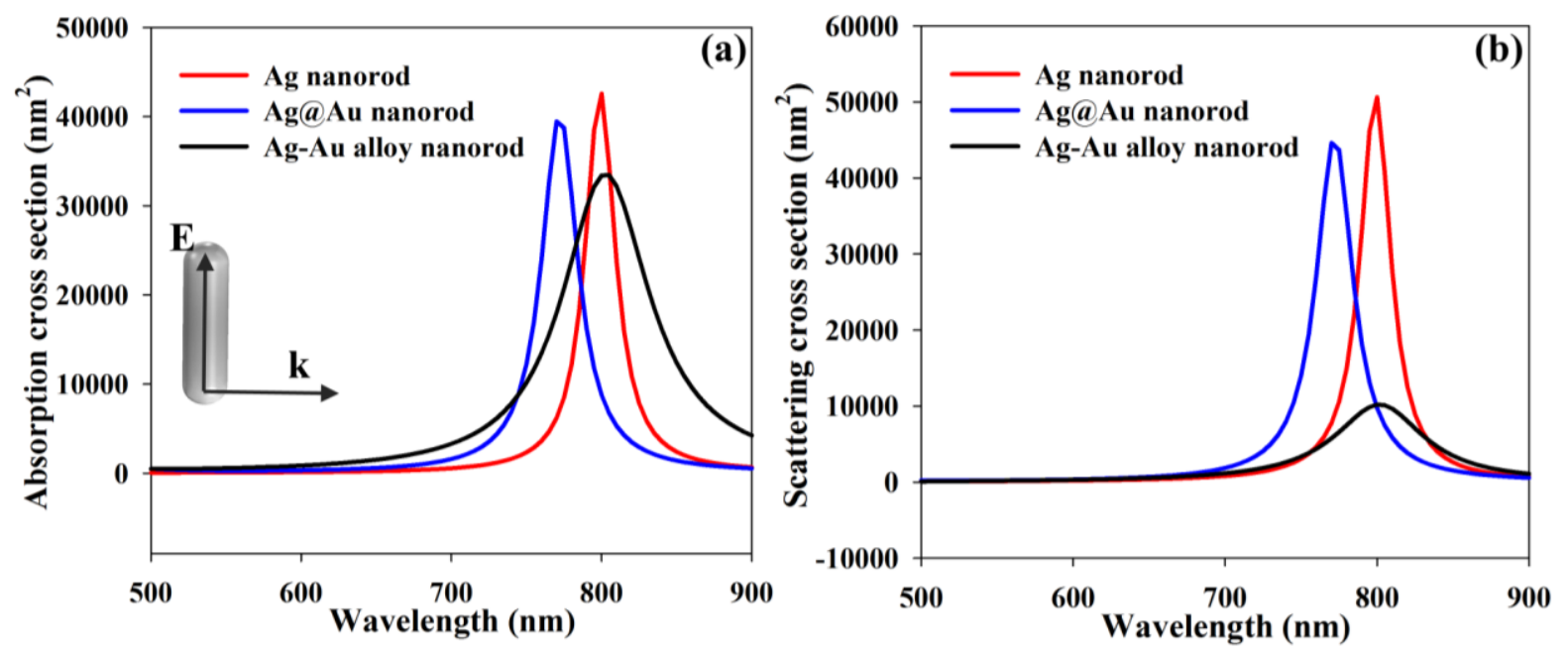

Figure 10. Effect of addition of $20 \%$ Au to an Ag nanorod (diameter: $20 \mathrm{~nm}$, aspect ratio: 4) as a thin shell or alloy.
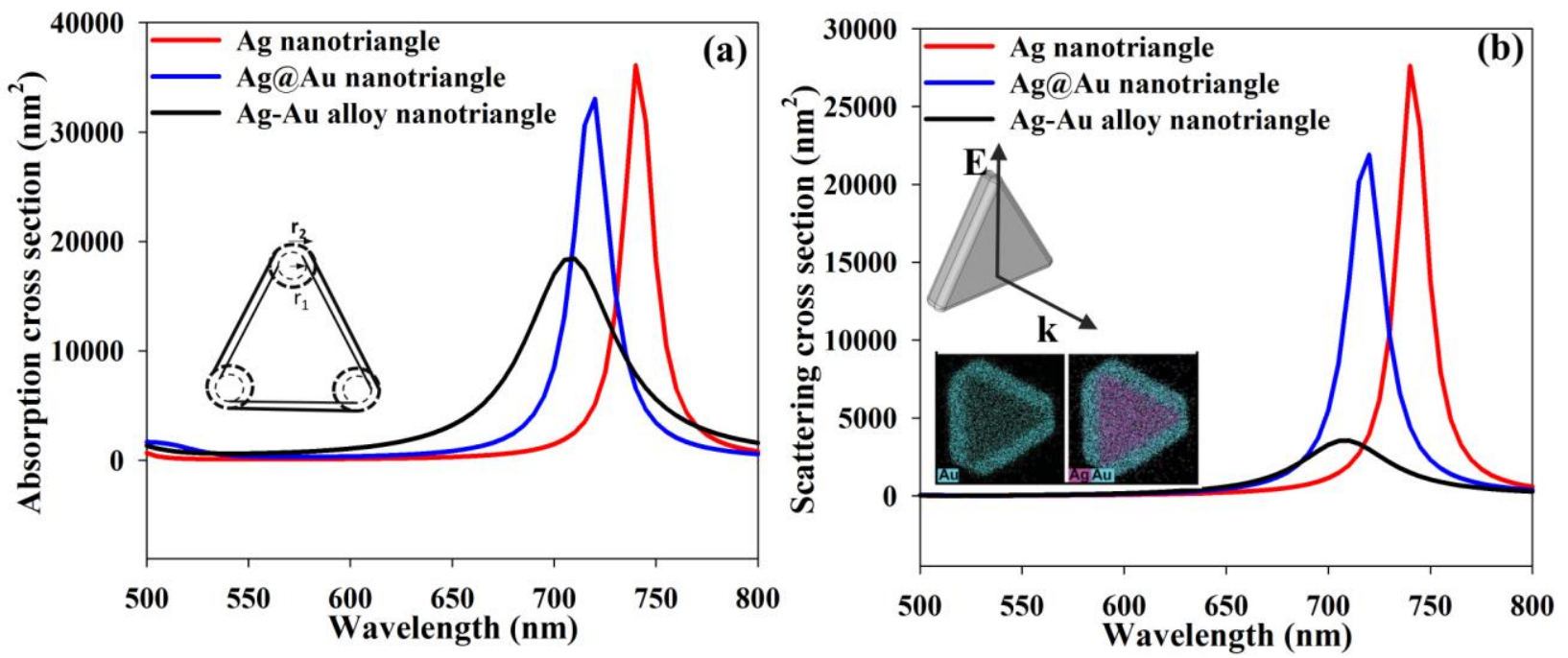

Figure 11. Effect of addition of $20 \% \mathrm{Au}$ to an $\mathrm{Ag}$ nanotraingle (60 nm each side, $7 \mathrm{~nm}$ thick) as a thin shell or alloy. Inset (a): schematic of a core-shell nanotriangle with corner curvatures $r_{1}$ and $r_{2}$ for the core and shell respectively. Inset (b): EDX mapping of synthesized Ag@ Au core-shell nanotriangle reproduced from reference ${ }^{16}$ with permission from Wiley-VCH (@ 2019). 

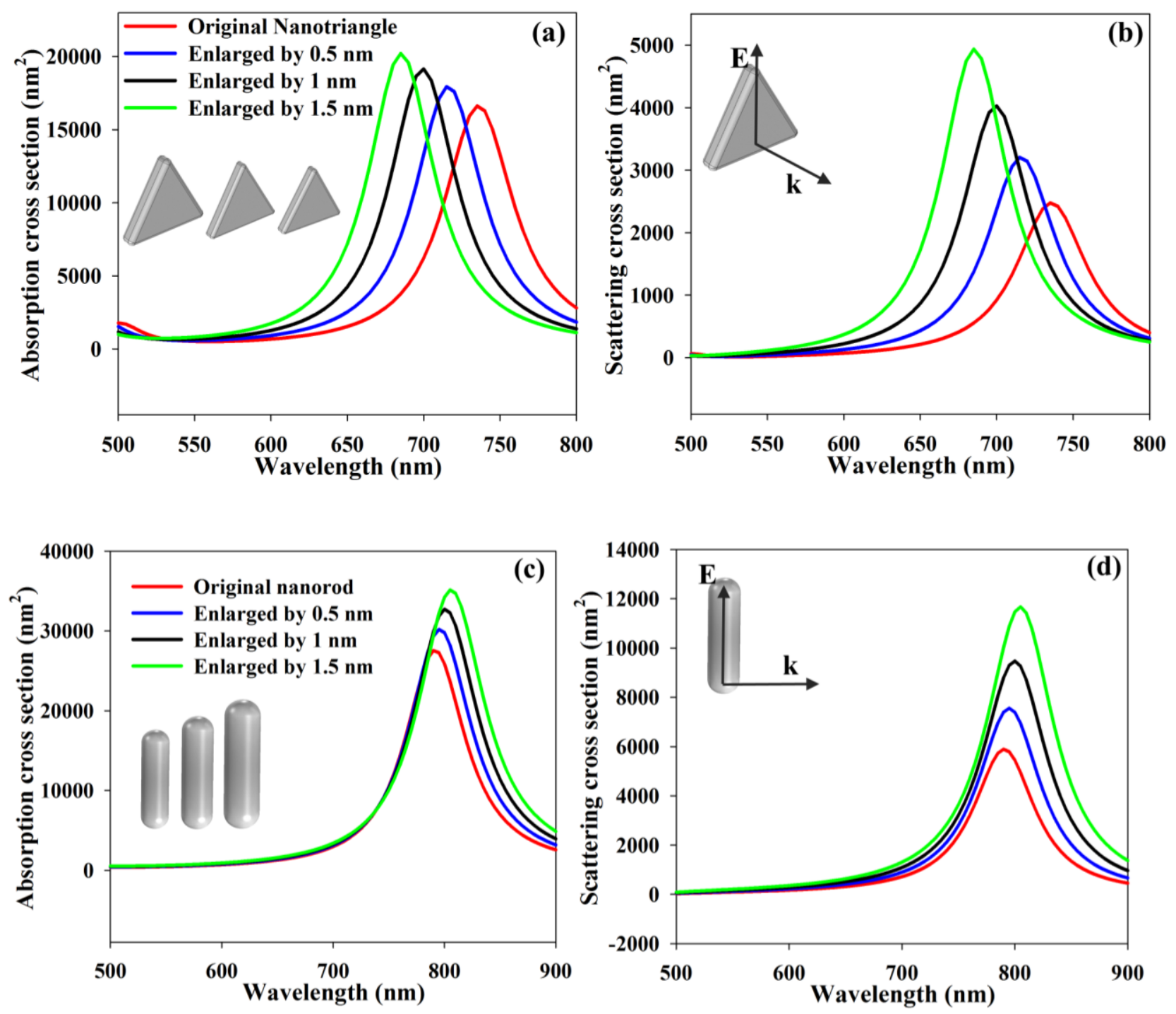

Figure 12. Effect of enlargement of $A u: A g=20: 80$ alloy nanotriangle $(a, b)$ and nanorod (c, d) by uniform addition of layers that reduces corner sharpness. Original Nanotriangle size: $\sim 60 \mathrm{~nm}$ each side, $7 \mathrm{~nm}$ thick; original nanorod size: $20 \mathrm{~nm}$ in diameter, $80 \mathrm{~nm}$ in length.

It is also useful to look further into the effect of alloying of nanorods and nanotriangles for a fixed volume, hereby excluding the geometrical effects. Figure 13 and Figure 14 compare the optical spectra of alloy nanorods and nanotriangles, respectively, showing that Ag exhibits both stronger absorption and scattering than $\mathrm{Au}$ in the infrared region. Interestingly, absorption and scattering intensities of alloy nanoparticles are 
lower than for both pure Ag and Au nanoparticles. Although the LSPR is red shifted for Au, the shift has been found to be rather discontinuous when moving from pure $\mathrm{Ag}$ to $\mathrm{Au}$.
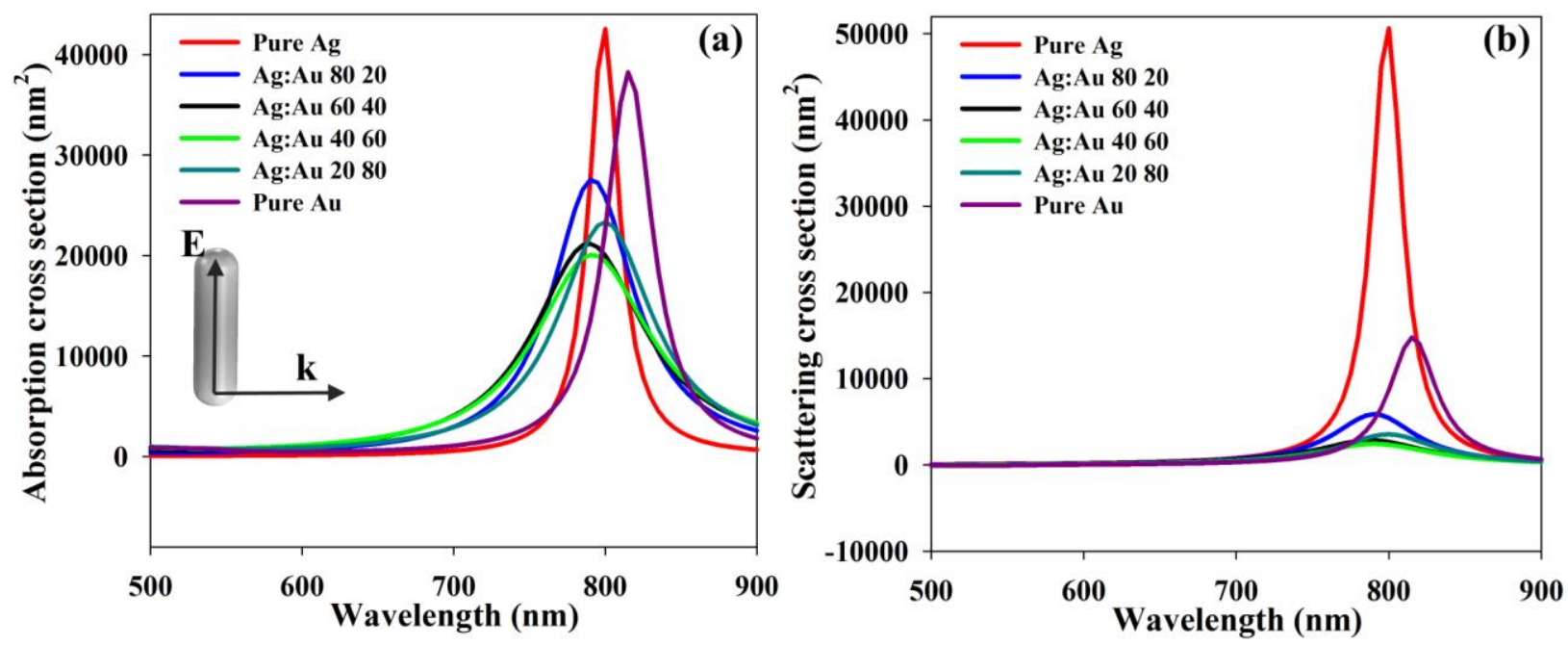

Figure 13. Absorption and scattering cross section of $\mathrm{Ag}-\mathrm{Au}$ alloy nanorods with varying composition and fixed size of diameter $20 \mathrm{~nm}$ and aspect ratio 4 .
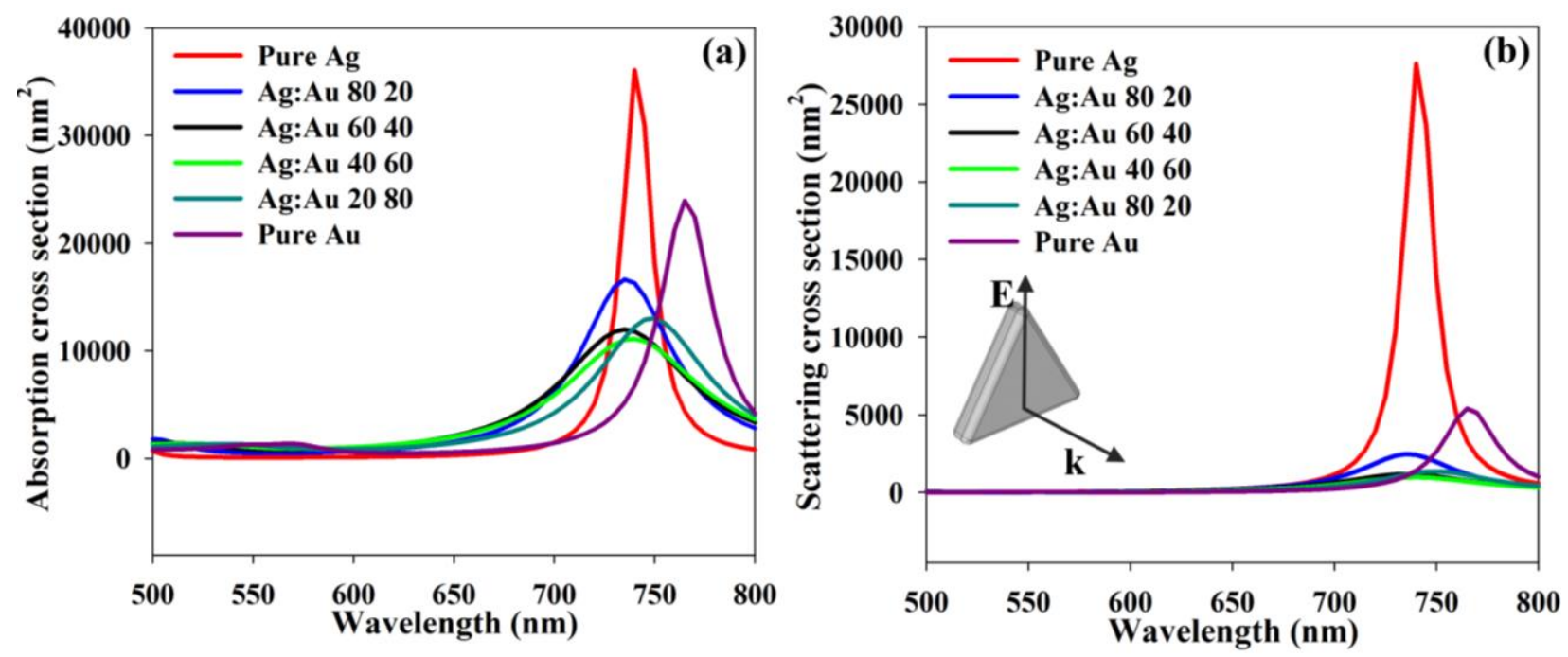

Figure 14. Absorption and scattering cross section of $\mathrm{Ag}-\mathrm{Au}$ alloy nanotriangles with varying composition and fixed size of $\sim 60 \mathrm{~nm}$ side length and $7 \mathrm{~nm}$ thickness.

The above analysis for anisotropic nanoparticles only considered the direction of the incident electric field to be parallel with the nanorod's axis i.e., longitudinal, while for nanotriangles the electric field is parallel 
to the triangular plane as shown in the Figures above. However, the alignment of the nanorods and nanotriangles with respect to the incident light also has an effect on the strength of the plasmonic enhancement. ${ }^{59,60}$ The intensity of the plasmonic response proportionally depends on the incident angle, i.e. it is maximal at $0^{\circ}$ and gradually decreases down to zero at $90^{\circ}$ incidence. ${ }^{59,60}$ Most importantly, the absolute position of the studied LSPR band is independent of the orientation in all cases; only the intensity changes and possibly addional bands may appear (e.g. the transversal LSPR band in the case of nanorods). Also, certain applications of anisotropic nanoparticles particularly exploit this orientation-dependence of the plasmonic response. ${ }^{60-62}$ It is useful to mention that the problem of orientation-dependent LSPR intensity in certain applications can be solved by using nanostars, which exhibit strong LSPR at large wavelengths, irrespective of their orientation. ${ }^{63}$

\subsubsection{Near-field enhancement}

In the absence of interband transition of $\mathrm{Au}$, it is also interesting to observe the near field characteristics of nanorods and nanotriangles in Figure 15. Clearly, due to edge effects, nanotriangles have significantly stronger near-field enhancement than nanorods. Overall, the optical cross sections in Figure 10 and 11 suggest that more "light" is captured by the nanorod. However, the sharper corners of the nanotriangles facilitates concentration of "light" over a smaller volume resulting in stronger near-field enhancement. So one must also keep in mind that uniform layer by layer enlargement of the nanorod will also strongly affect the near-field enhancement. It is clear from Figure 15 that the near-field enhancement is weakened by the addition of an $\mathrm{Au}$ shell to both the $\mathrm{Ag}$ nanorods and $\mathrm{Ag}$ nanotriangles, implying an overall reduction in plasmonic enhancement. Importantly, addition of $\mathrm{Au}$ as alloy weakens the near-field enhancement to a much greater extent. This along with the corresponding strong decrease in the scattering component (Figure 10 and 11) confirms a significant reduction in plasmonic excitation. 


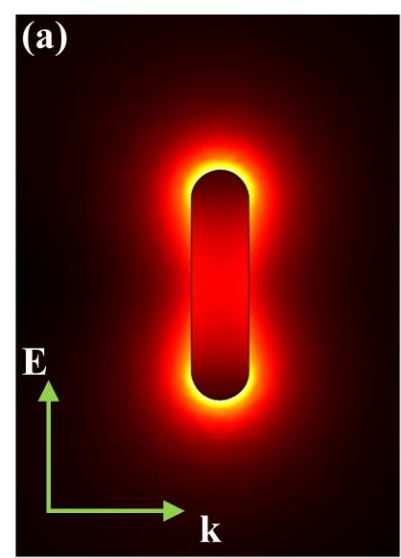

$800 \mathrm{~nm}$

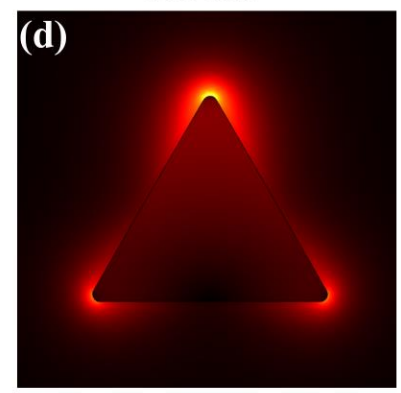

740 nm

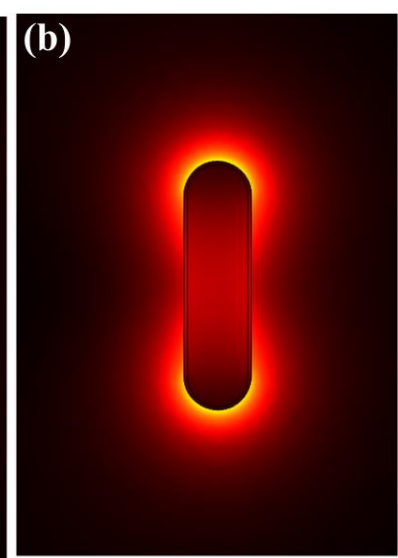

770 nm

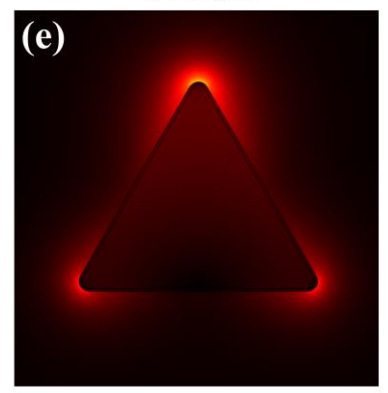

$720 \mathrm{~nm}$

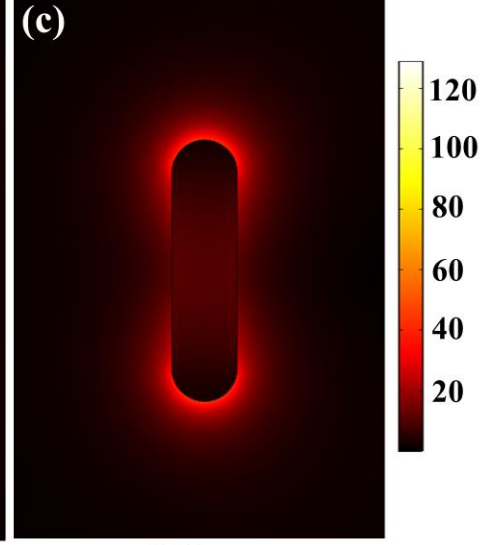

800 nm

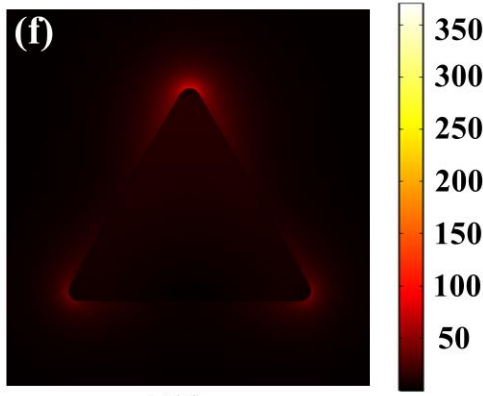

$710 \mathrm{~nm}$

Figure 15. Near field enhancement with respect to the incident radiation around nanords of (a) pure Ag (b) Ag@ Au (c) Alloy, and nanotriangles of (d) pure Ag (e) Ag@Au (f) Alloy. In correspondence to Figure 10 and 11, the Ag@ Au core-shell (b,e) and alloy (c,f) nanoparticles are modifications of pure Ag nanoparticles in (a, d) by addition of $20 \%$ $\mathrm{Au}$ as a thin shell or as alloy. Nanotriangle size: $\sim 60 \mathrm{~nm}$ each side, $7 \mathrm{~nm}$ thick; nanorod size: $20 \mathrm{~nm}$ in diameter, 80 $\mathrm{nm}$ in length.

\subsection{Photothermal Effect}

For spherical nanoparticles, while weakening of the optical response is generally disadvantageous for applications such as SERS, plasmonic phototcatalyis, etc.; the enhanced absorption and reduction of scattering due to Au incorporation is certainly advantageous for photothermal applications. ${ }^{64}$ Likewise, the widening of the spectra in the case of core-shell nanoparticles provides a wider window of usable wavelengths. A large $60 \mathrm{~nm} \mathrm{Ag} \mathrm{nanoparticle} \mathrm{with} \mathrm{high} \mathrm{radiative} \mathrm{loss} \mathrm{and} \mathrm{low} \mathrm{absorption} \mathrm{does} \mathrm{not} \mathrm{reach} \mathrm{a}$ maximum photothermal steady state temperature as high as a pure Au nanoparticle of the same size. The steady state temperature of $46.5^{\circ} \mathrm{C}$ of a $60 \mathrm{~nm}$ silver nanoparticle, Figure 16(a), is increased by $\sim 73 \%$ to 
$80.5^{\circ} \mathrm{C}$ simply by growing a thin gold shell of $2.14 \mathrm{~nm}$ around it, Figure $16(\mathrm{~b})$. Also the LSPR band does not exhibit any significant shift, Figure 9. The steady-state temperature for a $60 \mathrm{~nm}$ Au nanoparticle under the same irradiation conditions is low $\left(\sim 48.8^{\circ} \mathrm{C}\right.$ at $\left.435 \mathrm{~nm}\right)$, unless the incident wavelength is changed to $530 \mathrm{~nm}$ in order to achieve a similar steady temperature $\sim 80.7^{\circ} \mathrm{C}$, Figure $16(\mathrm{c}, \mathrm{d})$. Such enhancement of the photothermal response of $\mathrm{Ag}$ nanoparticles by the incorporation of a thin shell of $\mathrm{Au}$ is an interesting aspect as the chemical stabilization of Ag nanoparticles by $\mathrm{Au}$ in cellular environments has already been demonstrated. ${ }^{24}$ It is worth noting that the nanoparticle size is an important factor. For a smaller size of 20 $\mathrm{nm}, \mathrm{Ag}$ exhibits strong non-radiative decay of plasmons that leads to considerably higher absorption than $\mathrm{Au}$ (for which the absorption also has contributions from inter-band transitions). In this case, the temperature of $\mathrm{Ag}$ will be considerably higher than that of Au. Also, incorporation of $\mathrm{Au}$ in $\mathrm{Ag}$ in this case will reduce the absorption of pure Ag. However, due to smaller absolute absorption for smaller nanoparticles, the steady state temperature is in turn significantly lower than for large nanoparticles.

The disadvantages of silver nanoparticles in the context of biomedical applications are the LSPR being in the high frequency region and small bandwidth (FWHM $\sim 9 \mathrm{~nm}$ ). Thus, LSPR tuning of silver nanostructures over a wide wavelength range is important for their utility in different applications. This is enabled by anisotropic nanoparticles such as nanoplates, nanotriangles, nanorods, etc. ${ }^{58}$ Also, the absorption intensity of anisotropic nanoparticles at LSPR, as we have seen for nanorods and nanotriangles, is manifold higher than that for spherical nanoparticles. For comparison, Ag nanorods (diameter: $20 \mathrm{~nm}$, length: $80 \mathrm{~nm}$ ) and nanotriangles (60 $\mathrm{nm}$ each side, $7 \mathrm{~nm}$ thick) have $\sim 8.6$ and $\sim 7.3$ times the absorption cross section of Ag nanospheres $(60 \mathrm{~nm})$, respectively. For comparable sizes, Figure 17 shows that Ag nanotriangles and nanorods also have significantly higher photothermal steady state temperature than spherical nanoparticles. Since the same color map scale of Figure 16 is used in Figure 17 for direct comparison, one can see a much larger $>75{ }^{\circ} \mathrm{C}$ region around the nanotriangle and nanorod. In more detail, the steady state surface temperatures of an $\mathrm{Ag}$ nanorod and nanotriangle (Figure 17) amount to $\sim 294.3{ }^{\circ} \mathrm{C}$ and $\sim 258.5{ }^{\circ} \mathrm{C}$, respectively, compared to $\sim 46.5^{\circ} \mathrm{C}$ for an $\mathrm{Ag}$ nanosphere (Figure 16 (a)), which is attributed to a combined 
effect of a larger absorption component and smaller outer surface area for the silver nanorod and nanotriangle, when compared to the nanosphere in this study. It is also important to add that these temperatures are obtained under the assumption that no bubbles are forming due to boiling which, in reality, may influence the heat transfer characteristics. The boiling phenomenon is a more intricate aspect that requires separate treatment altogether and it is necessary to make the above assumptions to make meaningful comparisons. The implications of addition of $\mathrm{Au}$ as alloy or a protective layer around $\mathrm{Ag}$ nanorods and nanotriangles on the absorption are evident from Figure 10 and Figure 11. A thin protective layer of Au does not reduce the absorption greatly, implying a similar steady state temperature. On the other hand, addition as alloy results in a greater reduction in the absorption. However, the peak broadening provides a wider window of useful wavelengths.

Using the renewed physical insights from the present study, one may envisage the possibility of chemically stabilizing such anistropic Ag nanostructures with a thin gold shell. ${ }^{24}$ In view of the present trends of bimetallic nanoparticle synthesis and photothermal applications of nanoparticles, the present results open up new avenues for further investigation. 


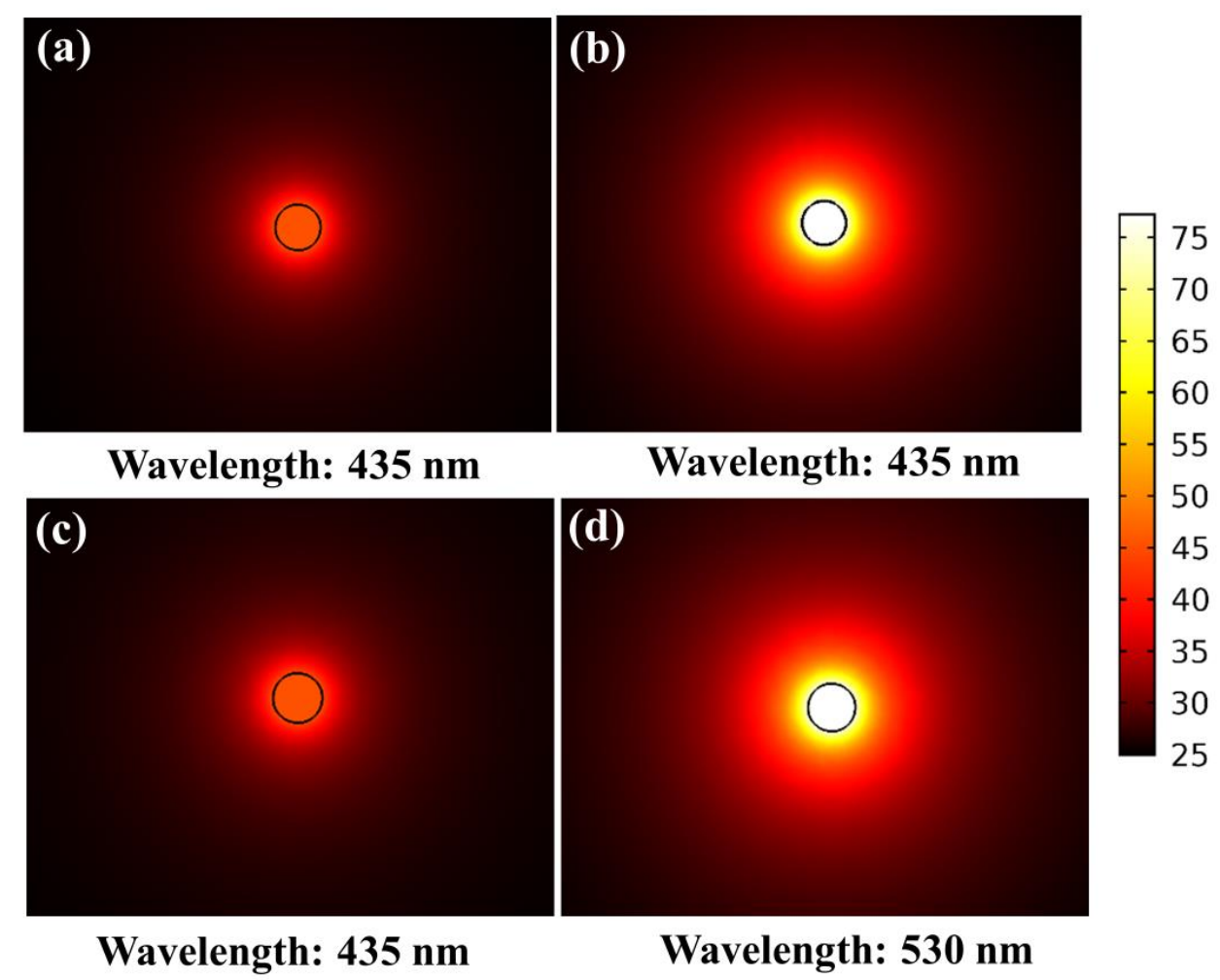

Figure 16. Steady state temperatures $\left({ }^{\circ} \mathrm{C}\right.$ ) of (a) $60 \mathrm{~nm} \mathrm{Ag}$ nanoparticle (b) $60 \mathrm{~nm} \mathrm{Ag}$ nanoparticle with a $2.14 \mathrm{~nm}$ thin Au shell (c,d) $60 \mathrm{~nm}$ Au nanoparticle. The intensity of incident radiation is $1 \mathrm{~mW} / \mu \mathrm{m}^{2}$ for all the cases.

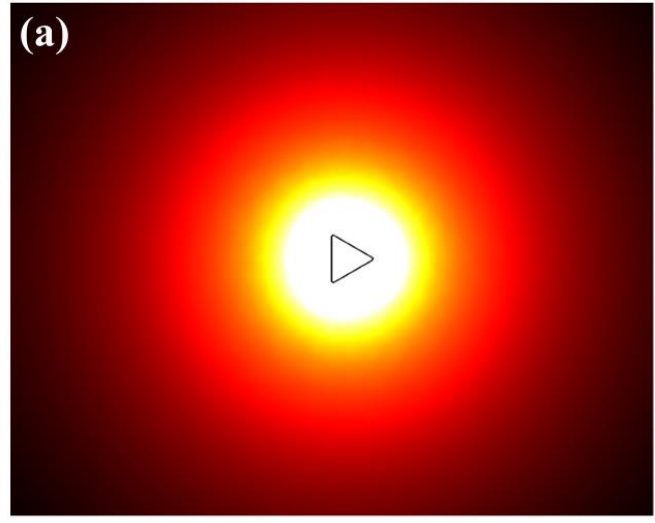

Wavelength: $740 \mathrm{~nm}$

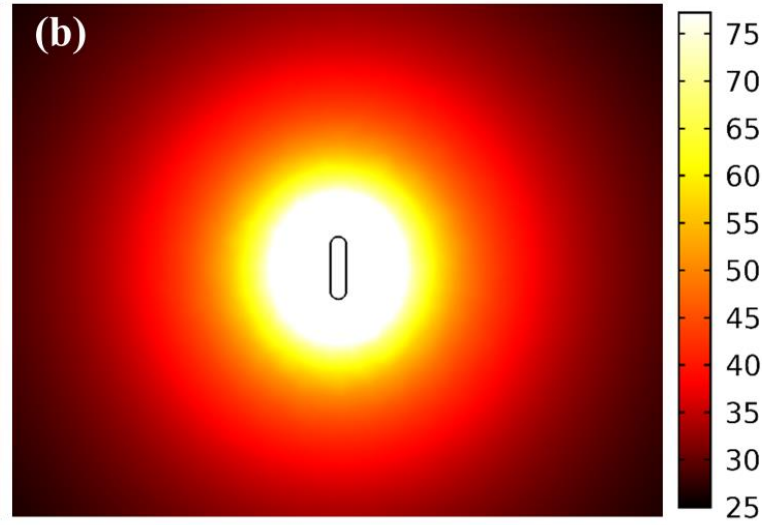

Wavelength: $800 \mathrm{~nm}$

Figure 17. Steady state temperatures $\left({ }^{\circ} \mathrm{C}\right)$ of an $\mathrm{Ag}$ nanotriangle $(\sim 60 \mathrm{~nm}$ each side, $7 \mathrm{~nm}$ thick) and $\mathrm{Ag}$ nanorod $(20$ $\mathrm{nm}$ in diameter, $80 \mathrm{~nm}$ in length). The intensity of incident radiation is $1 \mathrm{~mW} / \mu^{2}$ for all the cases. Note: the temperature scale is defined with respect to the nanospheres in Figure 16 to facilitate visual comparison between both. 


\section{Conclusion}

The present study compares the plasmonic behavior of Ag-Au alloy and core-shell bimetallic nanoparticles and shows the implications on the optical response and the photothermal effect. The comparison includes spherical as well as anisotropic nanoparticles (rods and triangles) to cover structures with and without contribution of intra-band transitions of $\mathrm{Au}$, respectively. The effects of bimetallic constitutions for these two cases are starkly different from each other.

For $60 \mathrm{~nm}$ spherical nanoparticles, the LSPR band shifts proportionally with changing composition in the alloy consititution; while for the core-shell constitution, the shift does not follow a proportional trend. Alloy nanoparticles display sharper spectral features with a characteristic LSPR peak in contrast to the widened spectra of core-shell nanoparticles. Incorporation of even a small amount of $\mathrm{Au}(20 \%)$ in $\mathrm{Ag}$ as alloy or core-shell significantly increases the absorption cross section due to interband transitions in Au. On the other hand, scattering is drastically reduced by the incorporation of the same amount of Au. Apart from the spectral characteristics, the optical intensities of alloy and core-shell nanoparticles are comparable. For nanorods and nanotriangles of comparable sizes, incorporation of $\mathrm{Au}$ in $\mathrm{Ag}$ results in a reduction of both absorption and scattering. While this reduction is not significant in a core-shell constitution, absorption and scattering are reduced dramatically in alloy nanoparticles. Especially, the drastic reduction in scattering suggests changes in the band structure by alloying that are detrimental to plasmonic enhancement. Also, unlike spherical particles, there is not a proportionate shift of the LSPR band with changing alloy composition from pure Ag to pure Au. Additionally for nanotriangles, the edge effects play a pivotal role in the LSPR intensity and spectral position. Thus, in anisotropic nanoparticles, geometrical effects become crucial while determining the plasmonic response. Increase in absorption and peak widening due to incorporation of $\mathrm{Au}$ in $\mathrm{Ag}$ improves the photothermal characteristics of $60 \mathrm{~nm}$ nanoparticles. In general, nanotriangles and nanorods are significantly better for photothermal applications due to much higher absorption in their optimal orientation. Addition of a thin Au shell around Ag nanorods or nanotriangles does not reduce the absorption intensities, thus the photothermal effect is well retained while the $\mathrm{Ag}$ 
structure is chemically stabilized. Thus, chemical protection of Ag nanoparticles by covering with an $\mathrm{Au}$ shell would be an efficient application strategy. These findings can serve as an input for the targeted synthesis of improved plasmonic nanomaterials. The numerical results and the obtained physical insights show interesting possibilities for the use and adaptation of bimetallic nanoparticles towards various plasmon-based applications.

\section{Acknowledgements}

R.B. acknowledges financial support for the University of Antwerp Special Research Fund (BOF) for a DOCPRO4 doctoral scholarship.

\section{Supporting information}

Comparison of optical properties from various literature, grid-independence test, extinction spectra and near-field enhancement simulations of selected cases.

\section{References}

(1) West, P. R.; Ishii, S.; Naik, G. V.; Emani, N. K.; Shalaev, V. M.; Boltasseva, A. Searching for Better Plasmonic Materials. Laser Photonics Rev. 2010, 4 (6), 795-808.

(2) Valenti, M.; Venugopal, A.; Tordera, D.; Jonsson, M. P.; Biskos, G.; Schmidt-Ott, A.; Smith, W. A. Hot Carrier Generation and Extraction of Plasmonic Alloy Nanoparticles. ACS Photonics 2017, 4 (5), 1146-1152.

(3) Mahmoud, M. A.; El-Sayed, M. A. Different Plasmon Sensing Behavior of Silver and Gold Nanorods. J. Phys. Chem. Lett. 2013, 4 (9), 1541-1545.

(4) Verbruggen, S. W.; Keulemans, M.; Goris, B.; Blommaerts, N.; Bals, S.; Martens, J. A.; Lenaerts, S. Plasmonic 'Rainbow' Photocatalyst with Broadband Solar Light Response for Environmental Applications. Appl. Catal. B Environ. 2016, 188, 147-153.

(5) Ristig, S.; Chernousova, S.; Meyer-Zaika, W.; Epple, M. Synthesis, Characterization and in Vitro Effects of $7 \mathrm{Nm}$ Alloyed Silver-Gold Nanoparticles. Beilstein J. Nanotechnol. 2015, 6 (1), 1212-1220.

(6) Qiu, G.; Ng, S. P.; Wu, C.-M. L. Bimetallic Au-Ag Alloy Nanoislands for Highly Sensitive Localized Surface Plasmon Resonance Biosensing. Sens. Actuators B Chem. 2018, 265, 459-467.

(7) Yang, Y.; Shi, J.; Kawamura, G.; Nogami, M. Preparation of Au-Ag, Ag-Au Core-Shell Bimetallic Nanoparticles for Surface-Enhanced Raman Scattering. Scr. Mater. 2008, 58 (10), 862-865.

(8) Link, S.; Wang, Z. L.; El-Sayed, M. A. Alloy Formation of Gold-Silver Nanoparticles and the Dependence of the Plasmon Absorption on Their Composition. J. Phys. Chem. B 1999, 103 (18), 3529-3533.

(9) Verbruggen, S. W.; Keulemans, M.; Martens, J. A.; Lenaerts, S. Predicting the Surface Plasmon Resonance Wavelength of Gold-Silver Alloy Nanoparticles. J. Phys. Chem. C 2013, 117 (37), 1914219145. 
(10) Verbruggen, S. W.; Keulemans, M.; Filippousi, M.; Flahaut, D.; Van Tendeloo, G.; Lacombe, S.; Martens, J. A.; Lenaerts, S. Plasmonic Gold-Silver Alloy on TiO2 Photocatalysts with Tunable Visible Light Activity. Appl. Catal. B Environ. 2014, 156-157, 116-121.

(11) Gilroy, K. D.; Ruditskiy, A.; Peng, H.-C.; Qin, D.; Xia, Y. Bimetallic Nanocrystals: Syntheses, Properties, and Applications. Chem. Rev. 2016, 116 (18), 10414-10472.

(12) Shankar, C.; Dao, A. T. N.; Singh, P.; Higashimine, K.; Mott, D. M.; Maenosono, S. Chemical Stabilization of Gold Coated by Silver Core-Shell Nanoparticles via Electron Transfer. Nanotechnology 2012, 23 (24), 245704.

(13) Sutter, E. A.; Sutter, P. W. In Situ Liquid Cell Electron Microscopy of Ag-Au Galvanic Replacement Reactions. Nanoscale 2017, 9 (3), 1271-1278.

(14) Yang, Y.; Liu, J.; Fu, Z.-W.; Qin, D. Galvanic Replacement-Free Deposition of Au on Ag for Core-Shell Nanocubes with Enhanced Chemical Stability and SERS Activity. J. Am. Chem. Soc. 2014, 136 (23), 8153-8156.

(15) Zhang, Z.; Bando, K.; Taguchi, A.; Mochizuki, K.; Sato, K.; Yasuda, H.; Fujita, K.; Kawata, S. AuProtected Ag Core/Satellite Nanoassemblies for Excellent Extra-/Intracellular Surface-Enhanced Raman Scattering Activity. ACS Appl. Mater. Interfaces 2017, 9 (50), 44027-44037.

(16) Liu, H.; Liu, T.; Zhang, L.; Han, L.; Gao, C.; Yin, Y. Etching-Free Epitaxial Growth of Gold on Silver Nanostructures for High Chemical Stability and Plasmonic Activity. Adv. Funct. Mater. 2015, 25 (34), 5435-5443.

(17) Blommaerts, N.; Vanrompay, H.; Nuti, S.; Lenaerts, S.; Bals, S.; Verbruggen, S. W. Unraveling Structural Information of Turkevich Synthesized Plasmonic Gold-Silver Bimetallic Nanoparticles. Small 0 (0), 1902791.

(18) Blommaerts, N.; Vanrompay, H.; Nuti, S.; Lenaerts, S.; Bals, S.; Verbruggen, S. W. Unraveling Structural Information of Turkevich Synthesized Plasmonic Gold-Silver Bimetallic Nanoparticles. Small 2019, 15 (42), 1902791.

(19) Wang, C.; Peng, S.; Chan, R.; Sun, S. Synthesis of AuAg Alloy Nanoparticles from Core/ShellStructured Ag/Au. Small 2009, 5 (5), 567-570.

(20) Shore, M. S.; Wang, J.; Johnston-Peck, A. C.; Oldenburg, A. L.; Tracy, J. B. Synthesis of $\mathrm{Au}($ Core)/Ag(Shell) Nanoparticles and Their Conversion to AuAg Alloy Nanoparticles. Small 2011, 7 (2), 230-234.

(21) Sanyal, U.; Davis, D. T.; Jagirdar, B. R. Bimetallic Core-Shell Nanocomposites Using Weak Reducing Agent and Their Transformation to Alloy Nanostructures. Dalton Trans. 2013, 42 (19), 7147-7157.

(22) Rioux, D.; Vallières, S.; Besner, S.; Muñoz, P.; Mazur, E.; Meunier, M. An Analytic Model for the Dielectric Function of Au, Ag, and Their Alloys. Adv. Opt. Mater. 2014, 2 (2), 176-182.

(23) Prodan, E.; Radloff, C.; Halas, N. J.; Nordlander, P. A Hybridization Model for the Plasmon Response of Complex Nanostructures. Science 2003, 302 (5644), 419-422.

(24) Espinosa, A.; Curcio, A.; Cabana, S.; Radtke, G.; Bugnet, M.; Kolosnjaj-Tabi, J.; Péchoux, C.; AlvarezLorenzo, C.; Botton, G. A.; Silva, A. K. A.; Abou-Hassan, A.; Wilhelm, C. Intracellular Biodegradation of $\mathrm{Ag}$ Nanoparticles, Storage in Ferritin, and Protection by a Au Shell for Enhanced Photothermal Therapy. ACS Nano 2018, 12 (7), 6523-6535.

(25) Liu, X.; Shan, G.; Yu, J.; Yang, W.; Ren, Z.; Wang, X.; Xie, X.; Chen, H.; Chen, X. Laser Heating of Metallic Nanoparticles for Photothermal Ablation Applications. AIP Adv. 2017, 7 (2), 025308.

(26) Cathcart, N.; Chen, J. I. L.; Kitaev, V. LSPR Tuning from 470 to $800 \mathrm{Nm}$ and Improved Stability of AuAg Nanoparticles Formed by Gold Deposition and Rebuilding in the Presence of Poly(Styrenesulfonate). Langmuir 2018, 34 (2), 612-621.

(27) Qi, Y.; Xing, T.; Zhao, J.; Weng, G.; Li, J.; Zhu, J.; Zhao, J. Tuning the Surface Enhanced Raman Scattering Performance of Anisotropic Au Core-Ag Shell Hetero-Nanostructure: The Effect of Core Geometry. J. Alloys Compd. 2019, 776, 934-947. 
(28) Kluczyk, K.; Jacak, W. Damping-Induced Size Effect in Surface Plasmon Resonance in Metallic NanoParticles: Comparison of RPA Microscopic Model with Numerical Finite Element Simulation (COMSOL) and Mie Approach. J. Quant. Spectrosc. Radiat. Transf. 2016, 168, 78-88.

(29) Borah, R.; Gupta, S.; Mishra, L.; Chhabra, R. P. Heating of Liquid Foods in Cans: Effects of Can Geometry, Orientation, and Food Rheology. J. Food Process Eng. 2020, e13420.

(30) Dasgupta, N.; Borah, R.; Mishra, P.; Gupta, A. K.; Chhabra, R. P. Combined Effects of Blockage and Yield Stress on Drag and Heat Transfer from an In-Line Array of Three Spheres. J. Dispers. Sci. Technol. 2019, 40 (6), 855-873.

(31) Donner, J. S.; Baffou, G.; McCloskey, D.; Quidant, R. Plasmon-Assisted Optofluidics. ACS Nano 2011, 5 (7), 5457-5462.

(32) Baffou, G.; Quidant, R.; García de Abajo, F. J. Nanoscale Control of Optical Heating in Complex Plasmonic Systems. ACS Nano 2010, 4 (2), 709-716.

(33) Chen, X.; Chen, Y.; Yan, M.; Qiu, M. Nanosecond Photothermal Effects in Plasmonic Nanostructures. ACS Nano 2012, 6 (3), 2550-2557.

(34) Mkhatshwa, M.; Motsa, S.; Sibanda, P. Overlapping Multi-Domain Spectral Method for Conjugate Problems of Conduction and MHD Free Convection Flow of Nanofluids over Flat Plates. Math. Comput. Appl. 2019, 24 (3), 75.

(35) Fukutani, H. Optical Constants of Silver-Gold Alloys. J. Phys. Soc. Jpn. 1971, 30 (2), 399-403.

(36) Ripken, K. Die optischen Konstanten von Au, Ag und ihren Legierungen im Energiebereich 2,4 bis 4,4 eV. Z. Für Phys. Hadrons Nucl. 1972, 250 (3), 228-234.

(37) Mulvaney, P. Surface Plasmon Spectroscopy of Nanosized Metal Particles. Langmuir 1996, 12 (3), 788-800.

(38) Etchegoin, P. G.; Le Ru, E. C.; Meyer, M. An Analytic Model for the Optical Properties of Gold. J. Chem. Phys. 2006, 125 (16), 164705.

(39) Etchegoin, P. G.; Le Ru, E. C.; Meyer, M. Erratum: “An Analytic Model for the Optical Properties of Gold” [J. Chem. Phys. 125, 164705 (2006)]. J. Chem. Phys. 2007, 127 (18), 189901.

(40) Nishijima, Y.; Akiyama, S. Unusual Optical Properties of the Au/Ag Alloy at the Matching Mole Fraction. Opt. Mater. Express 2012, 2 (9), 1226-1235.

(41) Peña-Rodríguez, O.; Caro, M.; Rivera, A.; Olivares, J.; Perlado, J. M.; Caro, A. Optical Properties of Au-Ag Alloys: An Ellipsometric Study. Opt. Mater. Express 2014, 4 (2), 403-410.

(42) Gong, C.; Leite, M. S. Noble Metal Alloys for Plasmonics. ACS Photonics 2016, 3 (4), 507-513.

(43) Patskovsky, S.; Bergeron, E.; Rioux, D.; Simard, M.; Meunier, M. Hyperspectral Reflected Light Microscopy of Plasmonic Au/Ag Alloy Nanoparticles Incubated as Multiplex Chromatic Biomarkers with Cancer Cells. Analyst 2014, 139 (20), 5247-5253.

(44) Palik, E. D. Handbook of Optical Constants of Solids, 1 edition.; Academic Press: San Diego, Calif., 1997.

(45) Zhang, A.-Q.; Qian, D.-J.; Chen, M. Simulated Optical Properties of Noble Metallic Nanopolyhedra with Different Shapes and Structures. Eur. Phys. J. D 2013, 67 (11), 231.

(46) Yang, Y.; Liu, J.; Fu, Z.-W.; Qin, D. Galvanic Replacement-Free Deposition of Au on Ag for Core-Shell Nanocubes with Enhanced Chemical Stability and SERS Activity. J. Am. Chem. Soc. 2014, 136 (23), 8153-8156.

(47) Johnson, P. B.; Christy, R. W. Optical Constants of the Noble Metals. Phys. Rev. B 1972, 6 (12), 43704379.

(48) Zheng, B. Y.; Zhao, H.; Manjavacas, A.; McClain, M.; Nordlander, P.; Halas, N. J. Distinguishing between Plasmon-Induced and Photoexcited Carriers in a Device Geometry. Nat. Commun. 2015, 6 (1), 1-7. 
(49) Li, X.-H.; Chou, J. B.; Kwan, W. L.; Elsharif, A. M.; Kim, S.-G. Effect of Anisotropic Electron Momentum Distribution of Surface Plasmon on Internal Photoemission of a Schottky Hot Carrier Device. Opt. Express 2017, 25 (8), A264-A273.

(50) Manjavacas, A.; Liu, J. G.; Kulkarni, V.; Nordlander, P. Plasmon-Induced Hot Carriers in Metallic Nanoparticles. ACS Nano 2014, 8 (8), 7630-7638.

(51) Huang, Y.; Lin, D.; Li, M.; Yin, D.; Wang, S.; Wang, J. Ag@Au Core-Shell Porous Nanocages with Outstanding SERS Activity for Highly Sensitive SERS Immunoassay. Sensors 2019, 19 (7), 1554.

(52) Gonzalez, C. M.; Martin, B.; Betancourt, T. Photochemical Synthesis of Bimetallic and Anisotropic Au-Containing Nanoparticles Using a One-Step Protocol. J. Mater. Chem. A 2014, 2 (41), 1757417585.

(53) Hodak, J. H.; Henglein, A.; Giersig, M.; Hartland, G. V. Laser-Induced Inter-Diffusion in AuAg Core-Shell Nanoparticles. J. Phys. Chem. B 2000, 104 (49), 11708-11718.

(54) Lim, D.-K.; Kim, I.-J.; Nam, J.-M. DNA-Embedded Au/Ag Core-Shell Nanoparticles. Chem. Commun. 2008, No. 42, 5312-5314.

(55) Banerjee, M.; Sharma, S.; Chattopadhyay, A.; Ghosh, S. S. Enhanced Antibacterial Activity of Bimetallic Gold-Silver Core-Shell Nanoparticles at Low Silver Concentration. Nanoscale 2011, 3 (12), 5120-5125.

(56) Rioux, D.; Vallières, S.; Besner, S.; Muñoz, P.; Mazur, E.; Meunier, M. An Analytic Model for the Dielectric Function of Au, Ag, and Their Alloys. Adv. Opt. Mater. 2014, 2 (2), 176-182.

(57) Albrecht, W.; Hoeven, J. E. S. van der; Deng, T.-S.; Jongh, P. E. de; Blaaderen, A. van. Fully Alloyed Metal Nanorods with Highly Tunable Properties. Nanoscale 2017, 9 (8), 2845-2851.

(58) Yang, L.; Zhou, Z.; Song, J.; Chen, X. Anisotropic Nanomaterials for Shape-Dependent Physicochemical and Biomedical Applications. Chem. Soc. Rev. 2019, 48 (19), 5140-5176.

(59) Ren, Y.; Qi, H.; Chen, Q.; Wang, S.; Ruan, L. Localized Surface Plasmon Resonance of Nanotriangle Dimers at Different Relative Positions. J. Quant. Spectrosc. Radiat. Transf. 2017, 199, 45-51.

(60) Fu, L.; Liu, Y.; Wang, W.; Wang, M.; Bai, Y.; Chronister, E. L.; Zhen, L.; Yin, Y. A Pressure Sensor Based on the Orientational Dependence of Plasmonic Properties of Gold Nanorods. Nanoscale 2015, 7 (34), 14483-14488.

(61) Chang, W.-S.; Ha, J. W.; Slaughter, L. S.; Link, S. Plasmonic Nanorod Absorbers as Orientation Sensors. Proc. Natl. Acad. Sci. 2010, 107 (7), 2781-2786.

(62) Biswas, S.; Nepal, D.; Park, K.; Vaia, R. A. Orientation Sensing with Color Using Plasmonic Gold Nanorods and Assemblies. J. Phys. Chem. Lett. 2012, 3 (18), 2568-2574.

(63) Gherman, A. M. M.; Boca, S.; Vulpoi, A.; Cristea, M. V.; Farcau, C.; Tosa, V. Plasmonic Photothermal Heating of Gold Nanostars in a Real-Size Container: Multiscale Modelling and Experimental Study. Nanotechnology 2020, 31 (12), 125701.

(64) Borah, R.; Verbruggen, S. W. Coupled Plasmon Modes in 2D Gold Nanoparticle Clusters and Their Effect on Local Temperature Control. J. Phys. Chem. C 2019.

\section{TOC Graphic}




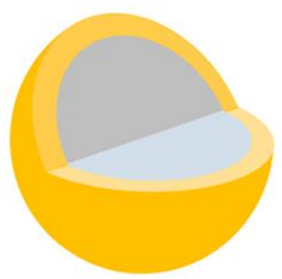

Ag@Au

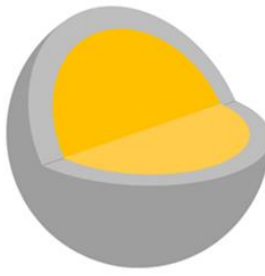

Au@Ag

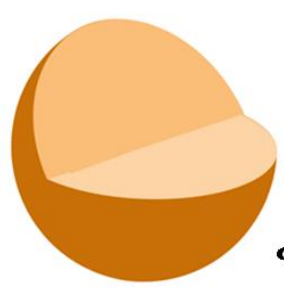

Alloy
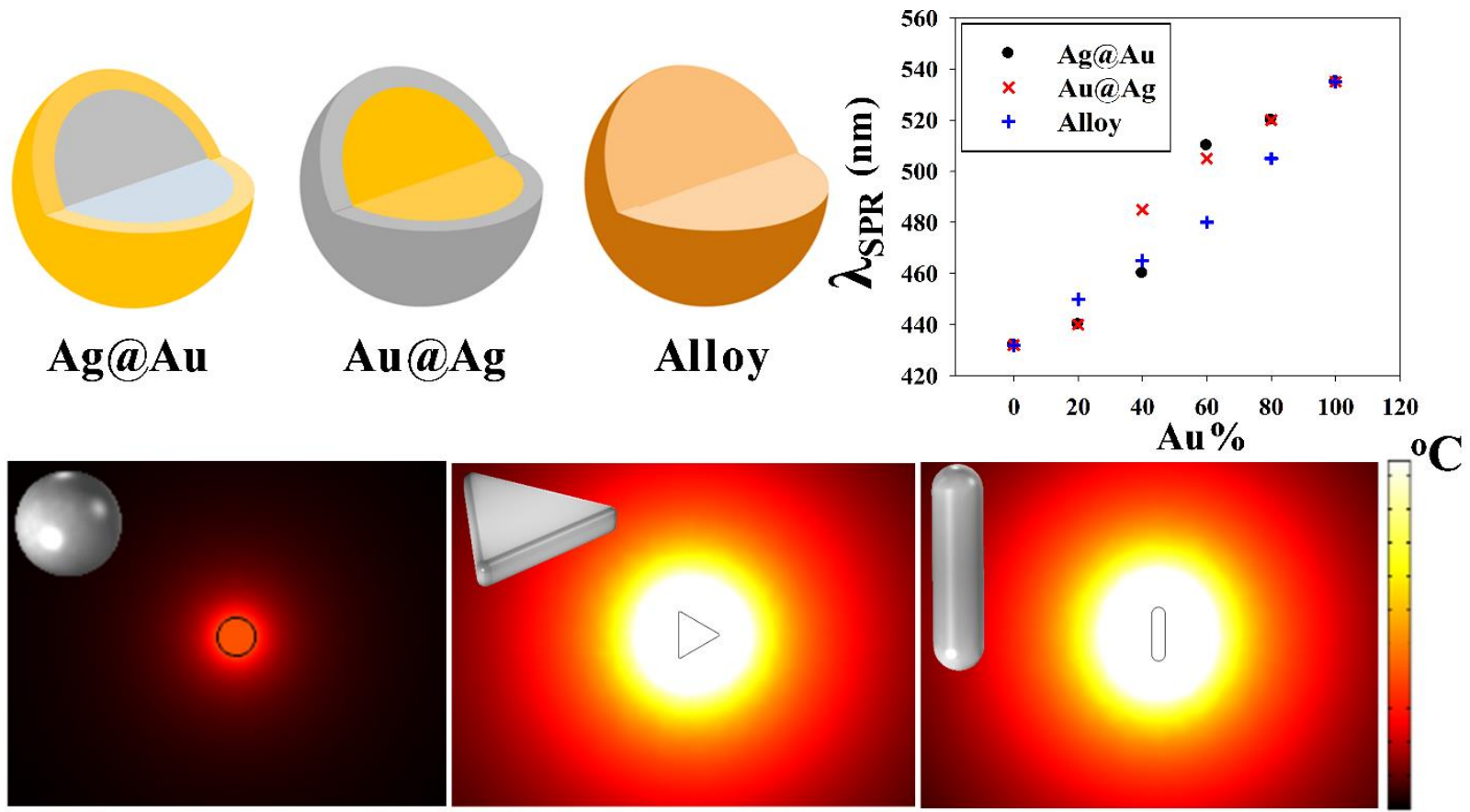